\title{
Two-Dimensional Infrared Correlation Spectroscopy, Conductor-like Screening Model for Real Solvents, and Density Functional Theory Study on the Adsorption Mechanism of Polyvinylpolypyrrolidone for Effective Phenol Removal in an Aqueous Medium
}

Muhammad Ammar Mohammad Alwi, Erna Normaya, Hakimah Ismail, Anwar Iqbal, Bijarimi Mat Piah, Mohd Armi Abu Samah, and Mohammad Norazmi Ahmad*

Cite This: ACS Omega 2021, 6, 25179-25192

Read Online

ACCESS

山ll Metrics \& More

回国 Article Recommendations

S1 Supporting Information

ABSTRACT: The discharge of industrial effluents, such as phenol, into aquatic and soil environments is a global problem due to its serious negative impacts on human health and aquatic ecosystems. In this study, the ability of polyvinylpolypyrrolidone (PVPP) to remove phenol from an aqueous medium was investigated. The results showed that a significant proportion of phenol (up to $74.91 \%$ ) was removed using PVPP at $\mathrm{pH}$ 6.5. Isotherm adsorption experiments of phenol on PVPP indicated that the best-fit adsorption was obtained using Langmuir models. The response peaks of the hydroxyl groups of phenol $(\mathrm{OH})$ and the carboxyl groups (i.e., $\mathrm{C}=\mathrm{O}$ ) of PVPP were altered, indicating the formation of a hydrogen bond between the PVPP and phenol during phenol removal, as characterized using 1D and 2D IR spectroscopy. The resulting complexes were successfully characterized based on their thermodynamic properties, Mulliken charge, and electronic transition using the DFT

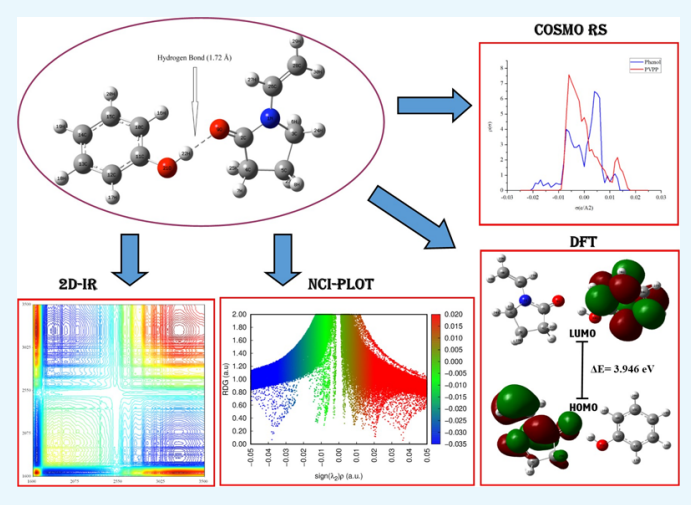
approach. To clarify the types of interactions taking place in the complex systems, quantum theory of atoms in molecules (QTAIM) analysis, reduced density gradient noncovalent interaction (RDG-NCI) approach, and conductor-like screening model for real solvents (COSMO-RS) approach were also successfully calculated. The results showed that the interactions that occurred in the process of removing phenol by PVPP were through hydrogen bonding (based on RDG-NCI and COSMO-RS), which was identified as an intermediate type $(\nabla 2 \rho(r)>0$ and $H<0$, QTAIM). To gain a deeper understanding of how these interactions occurred, further characterization was performed based on adsorption mechanisms using molecular electrostatic potential, global reactivity, and local reactivity descriptors. The results showed that during hydrogen bond formation, PVPP acts as a nucleophile, whereas phenol acts as an electrophile and the O9 atom (i.e., donor electron) reacts with the $\mathrm{H} 22$ atom (i.e., acceptor electron).

\section{INTRODUCTION}

Water pollution is of great concern to society, especially in urban areas. ${ }^{1}$ Water is a basic necessity of life and is essential to all living organisms. ${ }^{2}$ It is a critical factor that contributes to agricultural, socioeconomic, industrial, and technological development throughout the world, especially in developed countries. ${ }^{3}$ Although water is extremely important for life, the rapid development of household product manufacturing, petroleum refining, organic synthesis, pharmaceuticals, and agricultural industries across the world has contributed to increase levels of pollutants being discharged into the aquatic environment. ${ }^{4}$ The presence of various pollutants in water, including phenol, negatively impacts the ecosystem owing to their lethal effects. ${ }^{5}$

According to Anku et al., ${ }^{1}$ the global phenol product market in 2018 was worth USD 20 billion and is expected to increase to USD 28.4 billion by 2023 . This scenario will indirectly increase the amount of phenol discharged into aqueous systems as pollution. Phenol $\left(\mathrm{C}_{6} \mathrm{H}_{5} \mathrm{OH}\right)$ is an aromatic organic compound. It has been highlighted as one of the top 16 pollutants of concern discharged by the abovementioned industries by scientists and various policymakers (United States Environmental Protection Agency and the European Union). It is a concerning pollutant because it has both short-term and long-term toxicity effects in humans and animals when they are exposed to it via air, water, or direct contact. In addition, phenol easily dissolves in water,

Received: May 23, 2021

Accepted: September 9, 2021

Published: September 21, 2021 
a)

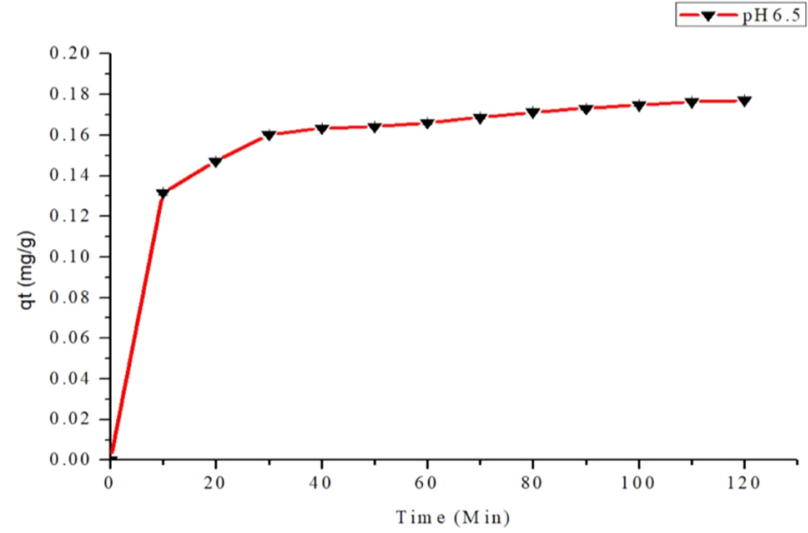

b)

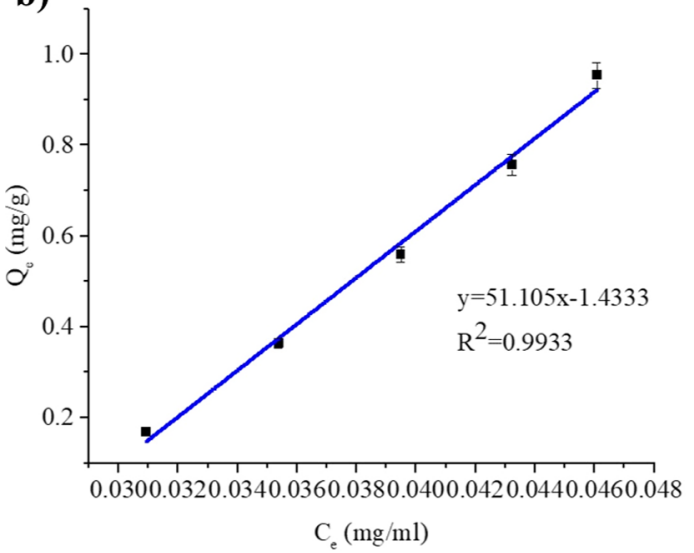

c)

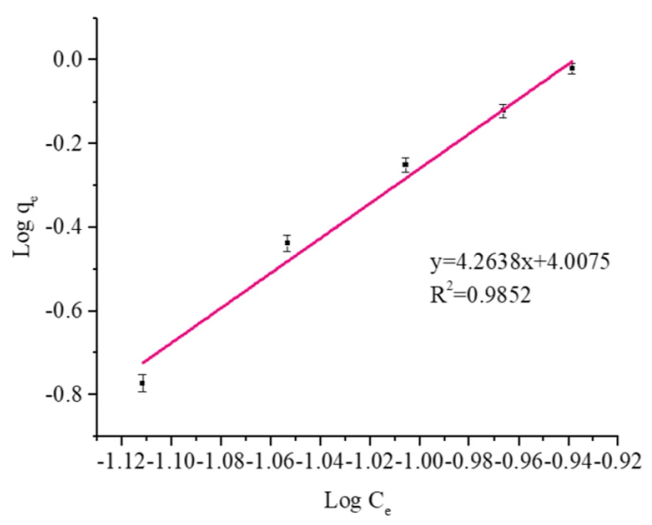

Figure 1. Phenol adsorption on PVPP at pH 6.5: (a) adsorption kinetic, (b) Langmuir isotherm, and (c) Freundlich isotherm.

which enables its wider application throughout the industry compared to other hydrocarbons.

Excessive phenol in the aquatic environment is not only undesirable and prohibited but also dangerous to humans and wildlife. ${ }^{6}$ Accordingly, researchers have developed a number of wastewater treatment techniques to treat effluent wastewater before it is discharged into the aquatic environment. ${ }^{7-9}$ Several methods have been used to remove phenol from wastewater. ${ }^{7,8}$ However, its adsorption based on polymers such as PVPP has received considerable interest because this method is easy to design and operate, is stable, and the surface of the polymer structure can be modified to increase the adsorption capacity and selectivity toward phenol pollutants. ${ }^{10-12}$ The manner in which PVPP reacts with (or binds to) phenol in water is critical to understand because the interaction between functional groups affects the adsorption process during phenol removal. In this respect, a theoretical estimation of the strength and nature of these interactions will help in explaining the mechanisms that result in experimental observations and could lead to the design of future PVPP-based adsorbents. ${ }^{13}$

Density functional theory (DFT) is one of the most common, efficient, and versatile tools in computational and theoretical chemistry for describing molecular interactions such as hydrogen bonding and van der Waals forces. ${ }^{14}$ Bai et al. ${ }^{15}$ reported the successful use of DFT in the analysis of hydrogen bond interactions during the removal of dimethylbenzoquinone from the atmosphere and aqueous environments using $\mathrm{TiO}_{2}$. Most previous PVPP research has focused on removing phenol from food and beverages. ${ }^{16}$ However, to our knowledge, no studies have investigated and integrated theoretical (RDG,
COSMO-RS, and DFT) and experimental (adsorption kinetics and isotherms, one-dimensional (1D) and two-dimensional (2D) infrared (IR) spectroscopy) approaches in explaining the adsorption mechanism of phenol removal from water systems. Therefore, the objective of this research was to study and evaluate the mechanism by which phenol adsorbs onto crospovidone and the efficiency of crospovidone in removing phenol from a water system by using spectroscopy and theoretical approaches.

In this study, phenol was chosen as the pollutant pollution of interest due to its substantial impact on the environment and human beings. ${ }^{1,6}$ The potential of PVPP as a phenol removal approach was, therefore, evaluated in a batch process using the adsorption isotherm and kinetic methods. To investigate and clarify the types of interactions involved and those which significantly contributed to the phenol adsorption efficiency of PVPP, several theoretical calculations were performed. DFT calculations using Gaussian 09 (Gaussian, Inc., USA) were used to identify the mode of hydrogen bond formation between the PVPP and phenolic compounds. Potential energy curves were also calculated to obtain the ideal conformation of the PVPPphenol complexes. All of the structures were then optimized to investigate the conformations developed during hydrogen bond formation. Chemical properties of the complex formed, such as its thermodynamic characteristics, Mulliken charge, and electronic transitions occurring in the system, were further characterized using the DFT approach. The quantum theory of atoms in molecules (QTAIM), conductor-like screening model for real solvents (COSMO-RS), and reduced density gradient noncovalent (RDG-NCI) plots were also used to investigate and 
identify the types of interactions in the PVPP-phenol complexes. In addition, global reactivity descriptors such as the highest occupied molecular orbital (HOMO), lowest occupied molecular orbital (LUMO), chemical potential, electronegativity, electrophilicity index, hardness, softness, and charge transfer were used to determine the relative stability and reactivity of PVPP and the phenolic compounds. Local chemical reactivity descriptors such as the Fukui function, local softness, and local hardness were further calculated to determine the active site of atoms (i.e., nucleophilic and electrophilic sites for hydrogen bond formation) in PVPP-phenol complexes.

\section{RESULTS AND DISCUSSION}

2.1. Adsorption Experiments. 2.1.1. Effect of $\mathrm{pH}$ Solutions. The $\mathrm{pH}$ of the solution plays a critical role in the removal of phenol from a water system because it can affect the charge of the adsorbent surface. ${ }^{17}$ The removal of phenol by PVPP was evaluated in solutions of $\mathrm{pH} 3.5$ and 6.5, while the initial concentration of phenol ions, contact time, and adsorbent amount were kept constant at $0.50 \mathrm{mg} / \mathrm{mL}, 90 \mathrm{~min}$, and $2.5 \mathrm{mg}$, respectively. The effect of PVPP on the water quality could be seen through color changes in the samples; the PVPP-treated and control samples $(0.5 \pm 0.01 \mathrm{mg} / \mathrm{mL}$ phenol concentration $)$ were clear and brown, respectively. The color change from slightly brown (before centrifugation) to white (PVPP-treated) suggested that the PVPP bound the phenol compounds. ${ }^{24}$ The results indicated that PVPP treatment significantly $(p<0.05)$ removed the phenol from the aqueous medium. Greater removal efficiency of phenol was recorded at $\mathrm{pH} 6.5$ (74.91 $\pm 0.53 \%)$ than at $\mathrm{pH} 3.5(66.54 \pm 0.41 \%)$. By optimizing the $\mathrm{pH}$, the percentage of phenol removal can be improved. As $\mathrm{pH} 6.5$ is close to the neutral condition of $\mathrm{pH} \mathrm{7,} \mathrm{an} \mathrm{electrostatic}$ interaction occurs between positive ions on the PVPP surface and phenolate ions, increasing the sorption. ${ }^{18}$ These findings were consistent with the results reported by Wei; ${ }^{17}$ these authors found that the $\mathrm{pH}$ of the medium can alter the charge distribution of both adsorbates and adsorbents. These alterations will subsequently affect the hydrogen bond interactions that contribute to the adsorption capacity.

2.1.2. Adsorption Kinetics. Figure 1a shows the kinetics curves for phenol adsorption on PVPP. The phenol adsorption rate was rapid until $20 \mathrm{~min}$, flattened between 20 and $50 \mathrm{~min}$, and finally reached the equilibrium state after $50 \mathrm{~min}$. This behavior can be attributed to a high surface area generated due to the foam conformation of the phenol-PVPP complexes. To gain a better understanding of the possible reaction mechanism of phenol on PVPP, the pseudo-first-order (PFO) model of eq 3 and pseudo-second-order (PSO) model of eq 4 were evaluated and compared. It is noted that from the determination coefficients $\left(R^{2}\right)$ and lower values of average relative errors (ARE), the PSO fits the experimental data better than the PFO for the adsorption period of phenol, which are PSO $\left(R^{2}: 0.9925\right.$ and ARE: $3.34 \%)$ and PFO ( $R^{2}: 0.9026$ and ARE: $\left.9.57 \%\right)$. Therefore, the sorption of phenol by PVPP follows the secondorder reaction kinetics, which supports the basic assumption in the model that chemisorption plays a major role in this adsorption system. ${ }^{18}$

2.1.3. Isotherm Study. In order to elucidate the adsorption mechanism of phenol removal using PVPP, the adsorption equilibrium was calculated based on two proposed isotherm models, the Langmuir and Freundlich models. The Langmuir isotherm model describes a monolayer adsorbent containing a finite number of adsorption sites with a homogeneous surface. In contrast, the empirical Freundlich isotherm model supposes that the adsorbent involves no uniform sorption and that the sorption of adsorbate occurs under a heterogeneous adsorption system. ${ }^{19}$ Figure 1b,c shows the Langmuir and Freundlich isotherm graphs. Based on the results in Table 1, the Langmuir

Table 1. Langmuir and Freundlich Model Constants for Adsorption of Phenol onto the PVPP at pH 6.5

\begin{tabular}{cll} 
model & parameters & \multicolumn{1}{c}{ values } \\
Langmuir & $q(\mathrm{mg} / \mathrm{g})$ & 0.2025 \\
& $b(\mathrm{~mL} / \mathrm{mg})$ & -18.1964 \\
Freundlich & $R^{2}$ & 0.9906 \\
& $K_{\mathrm{f}}(\mathrm{mg} / \mathrm{g})$ & $6.28 \times 10^{3}$ \\
& $n$ & 0.3473 \\
& $R^{2}$ & 0.9850
\end{tabular}

model gave a higher value for the correlation coefficient $\left(R^{2}\right.$ : 0.9905), which better described phenol adsorption onto PVPP compared to the Freundlich isotherm model $\left(R^{2}: 0.9850\right)$. In this regard, based on the review study by Issabayeva et al., ${ }^{20}$ many studies of phenol adsorption reported good fits of the phenol adsorption data in both isotherm models, indicating a mixed adsorption behavior.

In addition, the adsorption of phenol followed both models since the experimental equilibrium data showed a satisfactory fit to both models, and the values of data for each parameter obtained from the Freundlich isotherm model were found to be only slightly lower than those from the Langmuir isotherm model. Therefore, both parameters can fit the model isotherm, which signify that the phenol adsorption process on PVPP can be relatively heterogeneous. ${ }^{21}$ The uptake of phenol ions by PVPP was maximized at a concentration of $0.95 \mathrm{mg} / \mathrm{g}$. The obtained value of $q$ demonstrates that the PVPP can be used as an effective adsorbent for the removal of phenol from aqueous solution. The value of $n$ is less than 1 , showing that the sorption capacity is only decreased at lower equilibrium concentrations. The monolayer sorption model can be explained by a dimensionless constant $R_{\mathrm{L}}$ as being irreversible $\left(R_{\mathrm{L}}=0\right)$, favorable $\left(0<R_{\mathrm{L}}<1\right)$, linear $\left(R_{\mathrm{L}}=\right.$ should be greater than 1$)$, or unfavorable $\left(R_{\mathrm{L}}>1\right) .{ }^{20}$ Based on the separation factor $R_{\mathrm{L}}$ (Table 2 ), this method presents a favorable sorption condition for the

Table 2. $R_{\mathrm{L}}$ Value of the Langmuir Isotherm from the Adsorption of Phenol on the PVPP at $\mathrm{pH} 6.5$

$\begin{array}{cc}C_{0}(\mathrm{mg} / \mathrm{mL}) & \text { separation factor } R_{\mathrm{L}} \\ 0.5 & 0.0531 \\ 1.0 & 0.0273 \\ 1.5 & 0.0184 \\ 2.0 & 0.0138 \\ 2.5 & 0.0111\end{array}$

phenol ions, with $R_{\mathrm{L}}$ values of $0.0531-0.0111$ at $\mathrm{pH}$ 6.5. It can be simplified that by increasing the concentration of adsorbates, a higher adsorption capacity can be achieved.

2.1.4. 1D IR Analysis of the PVPP-Phenol Complex, PVPP, and Phenol. We investigated the main interaction that contributes to phenol removal by using 1D IR spectroscopy. Hydrogen bond interactions were identified as the main noncovalent interactions that contributed to PVPP-phenol complex formation during phenol removal. The two main functional groups involved during the hydrogen bond formation 
are the hydroxyl $(\mathrm{O}-\mathrm{H})$ group and the carbonyl group $(\mathrm{C}=\mathrm{O})$ in phenol and PVPP, respectively. Figure $2 \mathrm{a}$ shows the IR

a)

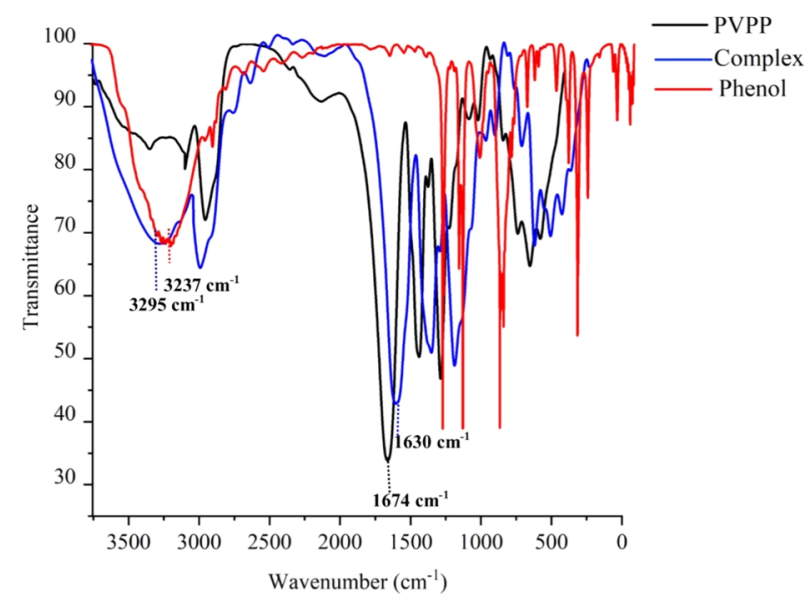

b)

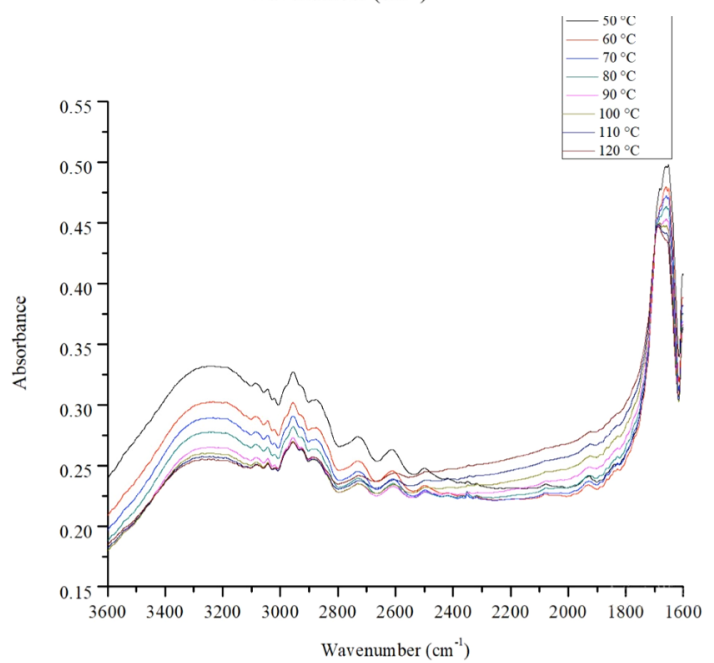

Figure 2. (a) 1D IR spectra of the PVPP-phenol complex, PVPP, and phenol and (b) absorption spectra of the PVPP-phenol complex from 3600 to $1600 \mathrm{~cm}^{-1}$ regions under different temperatures $\left(50-120^{\circ} \mathrm{C}\right)$.

spectra of the PVPP-phenol complex, PVPP, and phenol. The IR spectra of the monomers (phenol and PVPP) show absorption peaks at $3237 \mathrm{~cm}^{-1}$ (phenol) and $1674 \mathrm{~cm}^{-1}$ (PVPP), which were assigned to hydroxyl and carbonyl groups, respectively. However, in the IR spectrum of the PVPP-phenol complex, the wavenumbers of both absorption peaks $(\mathrm{O}-\mathrm{H}$ and $\mathrm{C}=\mathrm{O}$ ) were changed from 3237 to $3295 \mathrm{~cm}^{-1}$ and from 1674 to $1630 \mathrm{~cm}^{-1}$, respectively. This indicated the presence of a hydrogen bond interaction in the formation of the PVPPphenol complex. The wavenumber of the hydroxyl group (phenol) peak was increased due to the $\mathrm{O}-\mathrm{H}$ group becoming an electron acceptor, while that of the carboxyl group (PVPP) was decreased due to the $\mathrm{C}=\mathrm{O}$ group becoming an electron donor. This result is in agreement with Hook's law and the finding of $\mathrm{He}$ et al., ${ }^{22}$ which states that as the mass in the system increases, the wavenumber decreases and vice versa. ${ }^{23}$

2.1.5. 2D IR Correlational Analysis of the PVPP Complex. The temperature-dependent dynamic IR spectra of the PVPPphenol complex were obtained at temperatures from 50 to 120 ${ }^{\circ} \mathrm{C}$ at $10{ }^{\circ} \mathrm{C}$ intervals. This was carried out due to the ability of 2D IR to identify chemical classes that are altered during thermal perturbation. As illustrated in Figure $2 b$, all of the spectra were overlaid and baseline corrected in the region of 3600-1600 $\mathrm{cm}^{-1}$. The peak intensities and wavenumbers of the hydroxyl and carboxyl groups (at 3295 and $1630 \mathrm{~cm}^{-1}$, respectively) were sensitive to thermal perturbation when heat was applied. Figure $2 \mathrm{~b}$ shows that the intensities at 3295 and $1630 \mathrm{~cm}^{-1}$ were decreased when the temperature was increased. This is explained by the disruption of the hydrogen bond interaction that had formed in the PVPP-phenol complex. These results are supported by those of Popescu et al. ${ }^{24}$ and Sun et al. ${ }^{25}$ who respectively reported that the decreasing intensities of $\mathrm{OH}$ with $\mathrm{CH}$ and $\mathrm{OH}$ with $\mathrm{C}=\mathrm{O}$ were due to disrupted hydrogen bond interactions.

Two-dimensional correlation analysis allows overlapping spectral features that are not obvious in 1D spectra to be visualized because spectral resolution is enhanced by characterizing the dynamic variables of the data related to external perturbation. $^{26}$ Figure $3 \mathrm{a}, \mathrm{b}$ shows the synchronous and
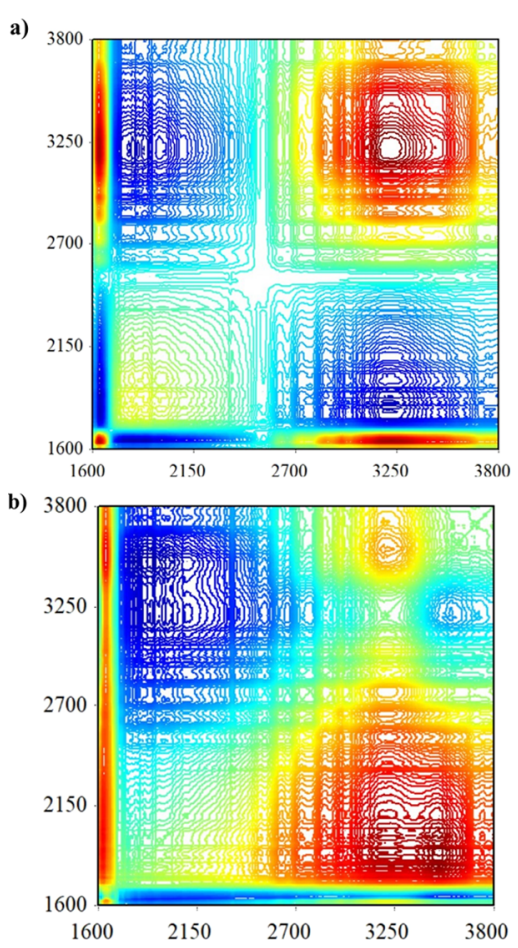

Figure 3. 2D IR spectroscopy of the PVPP-phenol complex in the region $3600-1600 \mathrm{~cm}^{-1}$ : (a) synchronous and (b) asynchronous.

asynchronous spectra of the 2D IR spectroscopy. The hydrogen bond stretching pattern for the PVPP-phenol complex can be observed in the $3600-1600 \mathrm{~cm}^{-1}$ region; each distinct carbonyl group and hydroxyl group in the PVPP-phenol complex causes a single stretching band at a certain wavenumber. The synchronous spectrum (Figure 3a) shows a cluster of 2D peaks (angle pattern) comprising two positive auto-peaks (1630 and $\left.3295 \mathrm{~cm}^{-1}\right)$ and two negative cross-peaks $(1630,3295)$ $\mathrm{cm}^{-1}$ and $(3295,1630) \mathrm{cm}^{-1}$. An auto-peak (a peak along the diagonal axis) shows the vibrational self-correlativity of certain functional groups at the same wavenumbers, and these two autopeaks in the spectrum $\left(1630\right.$ and $\left.3295 \mathrm{~cm}^{-1}\right)$ were assigned to $\mathrm{OH}$ and $\mathrm{C}=\mathrm{O}$, respectively. Cross-peaks (off-diagonal peaks) show the vibrational correlativity between two different functional groups, ${ }^{27,28}$ and they indicate that a hydrogen bond had formed. This is in accordance with the 1D IR spectroscopy results, where slight changes of the wavenumber (ascending and decreasing) occurred in the PVPP-phenol complex spectrum. 
Figure $3 \mathrm{~b}$ shows the asynchronous spectrum in $2 \mathrm{D}$ and $3 \mathrm{D}$ maps. The $2 \mathrm{D}$ asynchronous spectra are asymmetric with respect to the diagonal line in the correlation map. Unlike synchronous spectra, only off-diagonal cross-peaks, which can be negative or positive, are present in asynchronous spectra. The intensity of the asynchronous spectra $(\Psi(\nu 1, \nu 2))$ portrays sequential changes of spectral intensities obtained at $\nu 1$ and $\nu 2{ }^{29}$ The sign of the cross-peaks in the asynchronous spectra (two positive and two negative) gave important information about the order of reorientation for various transition dipole moments. In Figure 3b, the positive cross-peak ${ }^{26}$ is located in the lower-right section (rule 2), and the negative cross-peak is located in the upper-left section (rule 3). According to Noda's rule, ${ }^{29}$ this shows the transition dipole moment at the lower wavenumber $\nu 1\left(1630 \mathrm{~cm}^{-1}\right)$ reoriented before the transition moment at $\nu 2\left(3295 \mathrm{~cm}^{-1}\right)$. Thus, the following sequence of spectral intensity changes was obtained: $1630 \mathrm{~cm}^{-1}>3295$ $\mathrm{cm}^{-1}$.

2.2. Theoretical Results. 2.2.1. Potential Energy Curve (PES). In order to elucidate the interaction energy in the PVPPphenol complex, a PES, which is a first-step optimization was carried out to aid and determine the correct orientation of the molecular geometry (lowest minima energy). ${ }^{30}$ Figure S1 presents the PES of the hydrogen bond formation between phenol and PVPP. With the broadening and appearance of an asymmetrical single minimum, the PES of hydrogen bond ( $\mathrm{O}-$ $\mathrm{H})$ reveals a formation of a strong hydrogen bond (O9---H22O23) between PVPP and phenol. This result was supported by analyzing the shape of the curve; the strong hydrogen bond curve did not have a barrier that could block a proton from crossing between the donor and acceptor group during hydrogen bond formation. ${ }^{30,31}$ When the bond distance between the hydrogen of the hydrogen bond donor and the oxygen of the hydrogen bond acceptor fell below $1.7 \AA$, which is well within van der Waals contact for hydrogen and oxygen, the interaction energies rose sharply, as expected. We found that the interaction energy became higher when the bond distance became smaller $(1.5,1.6$, and $1.7 \AA$ gave $-67.977,-68.949$, and $-69.291 \mathrm{kcal} / \mathrm{mol}$, respectively). The repulsive part of the "electrostatic force" is caused by the Pauli exclusion principlewhen the atoms are pushed together too much, distortion of the orbitals will result in the electrons being "squished" out of the region between the atoms. The strong electrostatic repulsion between the two positively charged nuclei then pushes the atoms apart. $^{32}$ The minimum bond distance for the lowest interaction energy $(-69.329 \mathrm{kcal} / \mathrm{mol})$ was $1.7 \AA$. At this distance, the stabilization energy of the hydrogen bond was at the minimum because this conformation was the most stable conformation for this interaction. When the bond distance increased above $1.8 \AA$, it entered the vibration section and possessed high entropy energy. Subsequently, the dissociation energy increased because breakage of the hydrogen bond interaction required a high energy to overcome the bond's attractive energy. ${ }^{33}$

2.2.2. Molecular Geometry Optimization (Phenol and PVPP Monomers). Quantum chemical calculations have been shown to be outstanding predictors for determining the molecular structure of the inorganic and organic systems. These calculations are also useful for analyzing the noncovalent interaction especially the hydrogen bond. Thus, this study aimed to calculate the molecular geometry of a single isolated phenol and PVPP and the interactions between them in the solvent phase using a DFT/B3LYP method. In this study, the structures of the monomers (PVPP and phenol) and complex (PVPP- phenol) from the PES result were optimized. The optimized geometries of (a) PVPP, (b) phenol, and (c) the PVPP-phenol complex are shown in Figure S2. Selected comparative DFTcalculated bond angles and distances, measured during the formation of hydrogen bonds between PVPP and phenol groups, are given in Table S1. The effect of hydrogen bonding interactions on PVPP-phenol complex formation was investigated by comparing geometric parameters (bond distance and bond angle) for selected atoms in the monomers, PVPP, and phenols, with the atomic PVPP-phenol complex. The bond lengths of $\mathrm{C} 2=\mathrm{O} 9$ (monomer PVPP) and $\mathrm{O} 21-\mathrm{H} 22$ (monomer phenol) increased in the complex compared with those in the isolated molecules (Table S1). The bond lengths of $\mathrm{C} 2=\mathrm{O} 9$ (monomer PVPP) and O21-H22 (monomer phenol) were increased from 1.22 and $0.96 \AA$ to 1.24 and $0.99 \AA$ (complex), respectively. Interestingly, as shown in Table S1, the bond lengths $\mathrm{C} 2-\mathrm{C} 4, \mathrm{~N} 1-\mathrm{C} 2$, and $\mathrm{C} 11-\mathrm{O} 21$, which are located adjacent to the atom involved in hydrogen bond formation, decreased in the complex compared with those in the isolated molecules (PVPP and phenol). The bond lengths of $\mathrm{C} 2-\mathrm{C} 4, \mathrm{~N} 1-\mathrm{C} 2$, and $\mathrm{C} 11-\mathrm{O} 21$ were shortened from 1.52 , 1.39 , and $1.37 \AA$ to $1.51,1.37$, and $1.32 \AA$, respectively. In most cases, the bond length of the bond located adjacent to the atom will be shortened upon the formation of an intermolecular hydrogen bonding interaction. ${ }^{34}$ These results are consistent with those of Al-Ahmary et al., ${ }^{35}$ who reported an increment in the bond length of the bond involved in hydrogen bond formation and a shortening of the bond neighboring it.

It can be clearly seen in the optimized PVPP-phenol complex geometry (Figure S2c) that there has been an increase in the angles of $\mathrm{C} 4-\mathrm{C} 2=\mathrm{O} 9$ (PVPP monomer to complex) and C2O12-H13 (phenol monomer to complex) from 126.75 to $126.99^{\circ}$ and 110.787 to $111.75^{\circ}$, respectively, as the angles are facing each other. By contrast, the $\mathrm{N} 1-\mathrm{C} 2=\mathrm{O} 9$ angle decreases from $124.17^{\circ}$ (PVPP monomer) to $124.38^{\circ}$ (complex) owing to the increase in the $\mathrm{C} 4-\mathrm{C} 2=\mathrm{O} 9$ angle. In our opinion, the hydroxyl group $(\mathrm{O} 12-\mathrm{H} 13)$ in the phenol monomer is responsible for pushing the O9 atom (PVPP monomer) toward the N1 atom (PVPP monomer) and, subsequently, increasing the $\mathrm{C} 4-\mathrm{C} 2=\mathrm{O} 9$ angle (PVPP monomer). In this study, the hydroxyl groups that belong to the phenol moieties are brought close together during the formation of hydrogen bonds, and there is an associated cost in energy caused by overlapping electron clouds (Pauli and Born repulsion). This situation changes the conformation shape (angle) of the PVPP and the phenol monomer. In other words, this phenomenon is defined as a steric effect. ${ }^{36}$ The bond length and angle of the hydrogen bond (O9---H22-O21) are $1.72 \AA$ and $175.17^{\circ}$, respectively.

The hydrogen bond interactions in the optimized structures of phenol, the PVPP monomer, and the PVPP-phenol complex (belonging to the minimum potential energy curve or the most stable one), were verified by calculating the second derivative matrix (Hessien). This intermolecular hydrogen bonding interaction $\left(E_{\mathrm{H} \text { bond }}\right)$ was measured by defining the discrepancy in energy between the complex (containing the isolated monomer) and the BSSE value. ${ }^{37}$ The BSSE value must be calculated in order to identify the actual interactions of a hydrogen bond. ${ }^{38}$ According to geometrical, energetic, thermodynamic, and functional properties, the hydrogen bond strength is classified into three categories: weak $(<4 \mathrm{kcal} / \mathrm{mol})$, moderate $(4-15 \mathrm{kcal} / \mathrm{mol})$, and strong $(>15 \mathrm{kcal} / \mathrm{mol}){ }^{39}$ Therefore, based on Table S2, the interaction energy of this hydrogen bond (O9---H22-O21) is categorized as moderate 
$(10.114 \mathrm{kcal} / \mathrm{mol})$. In this study, the electrons involved in the formation of a hydrogen bond were transferred from an excited state (high potential energy) to a deeper potential well (potential energy $=0$ ) to obtain the most stable conditions for this interaction.

2.2.3. Thermodynamic Properties. The thermodynamic properties and relative energy values were calculated to analyze the chemical processes taking place in the PVPP-phenol complex. At $298.15 \mathrm{~K}$ and 1 atm pressure, the calculated thermodynamic parameters for the thermal, vibrational, and zero-point energies of the complex were $-164.76,162.982$, and $157.261 \mathrm{kcal} \mathrm{mol}^{-1}$, respectively (Table S3). The effect of temperature from 100 to $1000 \mathrm{~K}$ on the complex was also calculated using the B3LYP/6-311 ${ }^{++} \mathrm{G}(\mathrm{d}, \mathrm{p})$ level. Quadratic formulae were used to fit the correlation equations between heat capacity $\left(C_{\mathrm{p}, \mathrm{m}}^{0}\right)$, entropy $\left(S_{\mathrm{m}}^{0}\right)$, and enthalpy $\left(H_{\mathrm{m}}^{0}\right)$ changes and temperatures, with the corresponding fitting factors given by $\mathrm{R}^{2}$. Eqs $1-3$ and fitting factors are as follows:

$$
\begin{aligned}
C_{\mathrm{p}, \mathrm{m}}^{0} & =-4.03611+0.20166 \mathrm{~T}-7.460 \times 10^{-5} \times T^{2}\left(R^{2}\right. \\
& =0.9983) \\
H_{\mathrm{m}}^{0} & =155.656+0.01408 T+6.0828 \times 10^{-5} \times T^{2}\left(R^{2}\right. \\
& =0.9993) \\
S_{\mathrm{m}}^{0} & =57.30609+0.1877 T-2.8932 \times 10^{-5} \times T^{2}\left(R^{2}\right. \\
& =0.9994)
\end{aligned}
$$

The results (Figure 4 and Table S4) show that the heat capacity, enthalpy, and entropy all increase with the increasing

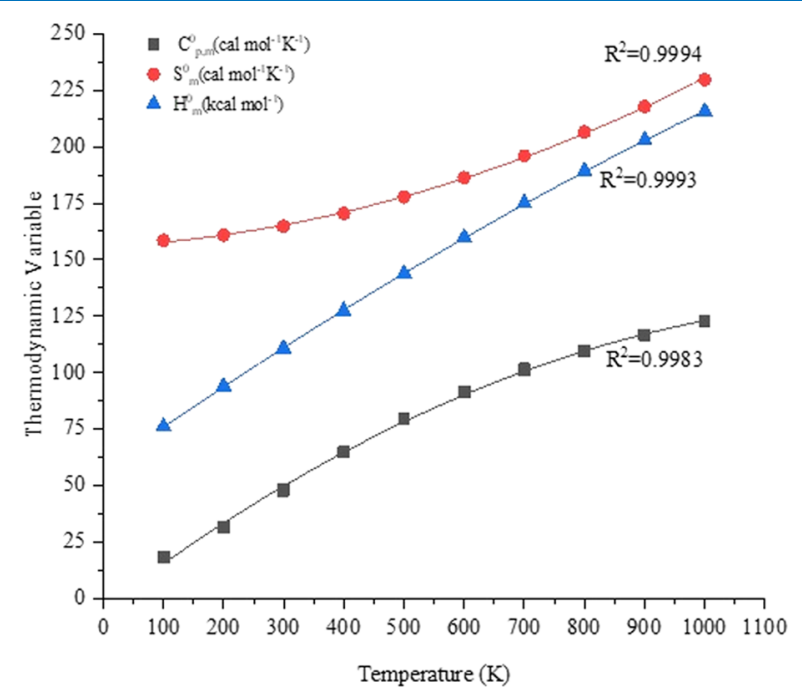

Figure 4. Correlation graph between thermodynamic variables and temperature.

temperature. ${ }^{40,41}$ The entropy and enthalpy of the complex changes demonstrated that the compound possesses more flexibility in changing its own thermodynamic system with the influence of temperature. ${ }^{42}$ These results clearly showed that the temperature strongly affects the chemical reactivity as well as the stability of the PVPP-phenol complex.

2.2.4. TD-DFT. The PVPP-phenol complex was further characterized using the time-dependent (TD)-DFT method. The results showed comparable experimental and theoretical spectra (Figure 5), indicating that the anticipated complex

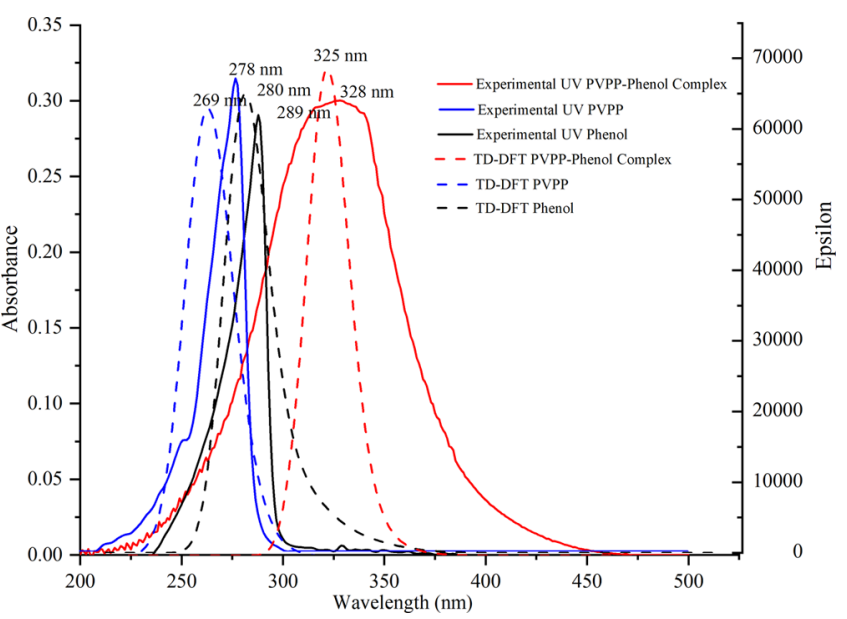

Figure 5. Experimental and calculated of UV spectra for the PVPPphenol complex, PVPP, and phenol

formation is in good agreement with experimental results. Further characterization was performed to analyze the types of electronic transitions, excitation energy, and assignments that occurred in the system, as shown in Table $3 .{ }^{43}$ In the experimental results, the PVPP and phenol absorbance peaks were observed at 278 and $289 \mathrm{~nm}$, respectively. Theoretical calculations found that the peaks occurred at 280 and $269 \mathrm{~nm}$, with contributions of $73.3 \%$ (HOMO-LUMO + 1) with assignment of the $n-\pi^{*}, \pi-\pi^{*}$ interactions for PVPP and $82.4 \%$ (HOMO- LUMO) with assignment of the $n-\pi, \pi-\pi^{*}$ interactions for phenol. The absorbance peak for the complex shifted to high wavelengths in both experimental $(328 \mathrm{~nm})$ and theoretical $(325 \mathrm{~nm})$ results, indicating that an interaction formed in the system caused by PVPP removing the phenol. The calculated result showed that $99 \%$ of the major contributions of the complex are from the HOMO-LUMO with its electronic transitions assigned as $n-\pi^{*}$ and $\pi-\pi^{*}$.

2.2.5. Charge Distribution Using Mulliken Atomic Charge (MAC) Analysis. To further study the interaction nature between the PVPP and phenol, charge analysis was calculated using the Mulliken method. The MACs calculated for the studied molecules (PVPP, phenol, and the PVPP-phenol complex) using the DFT/B3LYP $6-311^{++} \mathrm{G}(\mathrm{d}, \mathrm{p})$ polarized continuum model (PCM) method are shown in Figure S3; the atomic charge densities of the most significant variations are presented. Based on this study, the formation of an O9--- $\mathrm{H} 22$ O21 hydrogen bond (Figure S2) in the PVPP-phenol complex tends to increase the Mulliken atomic charges of selected involved atoms in complexed PVPP more than in the monomers (PVPP and phenol). When the PVPP-phenol complex is formed, the Mulliken atomic charge for atoms in PVPP and the phenol monomers increased for C4 $(-117.73$ to $-88.28 \mathrm{kcal} /$ $\mathrm{mol}), \mathrm{C} 11(-591.49$ to $-320.25 \mathrm{kcal} / \mathrm{mol}), \mathrm{O} 21(-369.39$ to $-362.99 \mathrm{kcal} / \mathrm{mol})$, and $\mathrm{H} 22(260.50-359.28 \mathrm{kcal} / \mathrm{mol})$. However, the Mulliken atomic charge decreased in monomer atoms for $\mathrm{N} 1(-30.08$ to $-38.54 \mathrm{kcal} / \mathrm{mol}), \mathrm{C} 2(178.76-22.32$ $\mathrm{kcal} / \mathrm{mol})$, and $\mathrm{O} 9(-383.63$ to $-376.16 \mathrm{kcal} / \mathrm{mol})$. This indicates that the Mulliken atomic charge is influenced by the length of the bond that forms between the atoms. ${ }^{44}$ The shorter the hydrogen bond distance, the higher the Mulliken atomic charge and vice versa. ${ }^{45}$ In addition, the 09 atom led to a more negative Mulliken atomic charge than $\mathrm{O} 21$ and $\mathrm{C} 2$, whereas $\mathrm{H} 22$ led to a more positive charge than $\mathrm{N} 1$ and $\mathrm{C} 4$, which may 
Table 3. Experimental and Calculated Electronic Transitions, Excitation Energy, and Their Assignments for the PVPP-Phenol Complex, PVPP, and Phenol

\begin{tabular}{|c|c|c|c|c|c|c|}
\hline \multirow[b]{2}{*}{ compound } & \multicolumn{2}{|c|}{ experimental } & \multicolumn{4}{|c|}{ theoretical } \\
\hline & $\lambda \max (\mathrm{nm})$ & excitation Energy (eV) & $\lambda \max (\mathrm{nm})$ & excitation energy $(\mathrm{eV})$ & major contribution $(<20 \%)$ & character \\
\hline \multirow[t]{2}{*}{ PVPP-phenol complex } & 328 & 3.78 & 325 & 3.81 & 99\% (HOMO-LUMO) & $n-\pi^{*}$ \\
\hline & & & & & & $\pi-\pi^{*}$ \\
\hline \multirow[t]{4}{*}{ PVPP } & 278 & 4.46 & 280 & 4.43 & 73.30\% (HOMO-LUMO + 1) & $n-\pi^{*}$ \\
\hline & & & & & & $\pi-\pi^{*}$ \\
\hline & & & & & 24\% (HOMO-LUMO) & $\mathrm{n}-\pi$ \\
\hline & & & & & & $\pi-\pi^{*}$ \\
\hline \multirow[t]{2}{*}{ phenol } & 289 & 4.29 & 269 & 4.61 & 82.4\% (HOMO-LUMO) & $\mathrm{n}-\pi$ \\
\hline & & & & & & $\pi-\pi^{*}$ \\
\hline
\end{tabular}

Table 4. Topology Analysis of the PVPP-Phenol Complex (Electron Density $(\rho \mathrm{BCP})$, Laplacian of Electron Density $\nabla 2 \rho(r)$, Kinetic Energy Density $G(r)$, Potential Energy Density $V(r)$, and Total Energy Density $H(r))$ at the Bond Critical Point $(\text { BCP })^{\text {a }}$

$\begin{array}{ccccccc}\text { interactions } & \mathrm{BD} & \rho(r) & \nabla 2 \rho(r) & G(r) & H(r) & \mathrm{DI}(A, B) \\ \mathrm{C} 2 \mathrm{O} 9-\mathrm{O} 21 \mathrm{H} 22 & 1.72 & +0.0381 & +0.0952 & +0.0336 & -0.0435 & -0.0099 \\ a \rho(r), \nabla 2 \rho(r), G(r), V(r), \text { and } H(r) & \text { in a.u. and } V(r) \text { in brackets }(\mathrm{kJ} / \mathrm{mol}) . & & & \end{array}$

be attributed to differences in electron polarizability. The Mulliken atomic charge of the $\mathrm{O} 9$ atom is larger than that of $\mathrm{H} 22$ and has a more easily polarizable electronic cloud, making electron donation from $\mathrm{O} 9$ to $\mathrm{H} 22$ more efficient for hydrogen bond formation. In this study, we focused on the amount of energy associated with the Mulliken atomic charges of atoms involved in the hydrogen bond formation.

2.2.6. Hydrogen Bond Interaction Analysis. 2.2.6.1. QTAIM Analysis. The quantum theory of atoms in molecules (QTAIM) is a useful tool to identify the intra- and intermolecular hydrogen bond and to study the bond structure of molecular systems. ${ }^{46}$ In this analysis, the nature of interactions such as covalent, weak electrostatic, etc. between the atoms can be characterized through several topological parameters like electron densities $(\rho \mathrm{r})$ and their Laplacian $(\nabla 2 \rho(r))$, the local potential electron energy density $(\mathrm{Vr})$, kinetic electron density $(\mathrm{Gr})$, and the total electron energy densities $(\mathrm{Hr})$ at the bond critical points (BCP). ${ }^{47,48}$ At BCP, the nature of the hydrogen bond can be classified by this hypothesis: (i) values of $(\nabla 2 \rho(r))<0, H<0$, are associated with a strong hydrogen bond; (ii) values of $(\nabla 2 \rho(r))>0, H<0$, signify intermediate-type hydrogen bonds; (iii) values of $(\nabla 2 \rho(r))>0, H>0$, are associated with weak hydrogen bond interactions; where $H(r)$ is also known as the summation of $G(r)+V(r){ }^{46,47}$ The molecular graphs of PVPP and phenol using the AIMAII program is shown in Figure S4. It was identified that the interacting atoms of the hydrogen bonds happened between $\mathrm{C} 2 \mathrm{O} 9--\mathrm{O} 21 \mathrm{H} 22$. Thus, the topological parameters of these interacting atoms are listed in Table 4. Based on the parameters, it can be indicated that the hydrogen bond between PVPP and phenol is intermediate-type hydrogen bonds since $\nabla 2 \rho(r)>0, H<0,[(\nabla 2 \rho(r))=+0.0952$ a.u., $H=-0.0099$ a.u.]. Meanwhile, the electron density $(\rho)$ and the Laplacian of electron density $\nabla 2 \rho(r)$ of the intermolecular interaction C2O9--O21H22 are +0.0381 a.u. and +0.0952 a.u., respectively; the positive Laplacian indicates that the interactions exhibit a closed-shell interaction.

2.2.6.2. RDG-NCI Approach. RDG-NCI analysis is one of the modeling methods to investigate the type of noncovalent interactions that occurred in the system. The interactions predicted to be involved during the removal of phenol from the aqueous mixture were evaluated and characterized using the RDG-NCI program. As shown in Figure 6a, there are three types a)

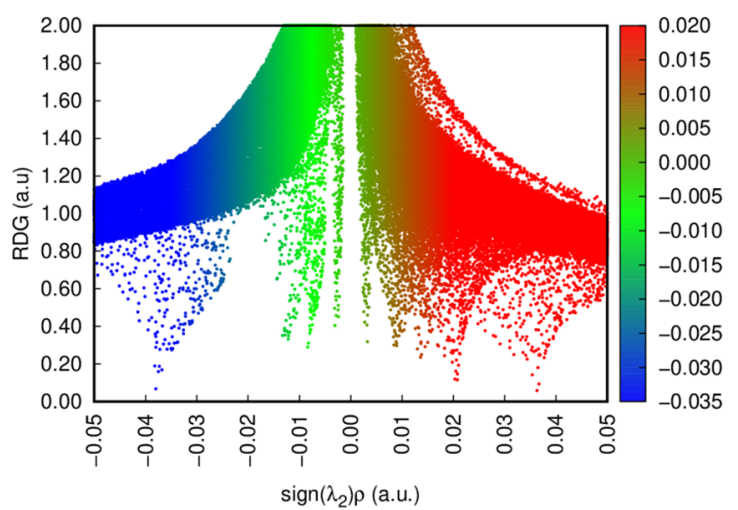

b)

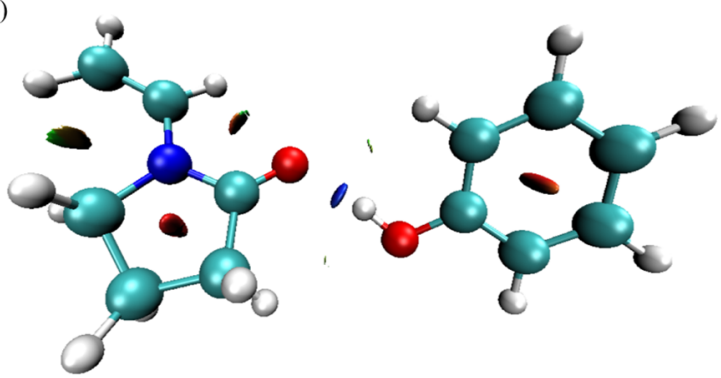

Figure 6. (a) NCI-RDG scatter diagrams of the PVPP-phenol complex and (b) color-mapped RDG isosurface graph.

of noncovalent interactions involved during PVPP-phenol complex formation, namely, steric effects (red; $\lambda_{2}>0$ ), hydrogen bonds (blue; $\lambda_{2}<0$ ), and van der Waals bonds (green; $\lambda_{2}=0$ ). However, hydrogen bonding interactions (an intermolecular interaction) play the primary role in phenol removal. These interactions occurred between the $\mathrm{C}=\mathrm{O}$ (PVPP) and $\mathrm{OH}$ (phenol) groups (Figure 6b). This interaction was computed from the most stable PVPP-phenol complex taken from the optimization result. The other interactions, namely, steric effects (in the PVPP and phenol ring) and van der Waals interactions (between $\mathrm{CH}_{2}$ and $\mathrm{CH}_{2} \mathrm{~N}$ in PVPP, i.e., intramolecular) can be seen in Figure $6 \mathrm{~b}$. This finding supported the DFT results, which confirmed that the hydrogen bond interactions play a primary role during phenol removal from the aqueous mixture. 
2.2.6.3. COSMO-RS Approach. COSMO-RS is a method that can be used to predict and identify the molecular interaction during phenol removal using PVPP. The interaction mechanism between PVPP and phenol can be explained using a $\sigma$-profile and a $\sigma$-potential. Based on the COSMO-RS theory, the $\sigma$-profile and the $\sigma$-potential are divided into three types of interaction regions, which are the hydrophobic $(-0.0084 \mathrm{e} / \mathrm{A} 2>\sigma<0.0084$ e/A2) and hydrogen bond donor (HBD; $\sigma<-0.0084$ e/A2) and hydrogen bond acceptor (HBA; $\sigma>0.0084$ e/A2). ${ }^{49}$ Furthermore, the $\sigma$-potential can be used to evaluate the affinity of the molecules toward solvents in aqueous solution based on their hydrogen bonding and polarity. Typically, the negative and positive values of the $\sigma$-potential (1/A2) illustrate the stronger affinity and repulsion, respectively, of a molecule toward the solvents. ${ }^{50}$ In this study, COSMO-RS has been used to elucidate the interaction mechanism during the phenol removal by using the PVPP adsorbent. In Figure 7a, PVPP has a small peak in the

a)

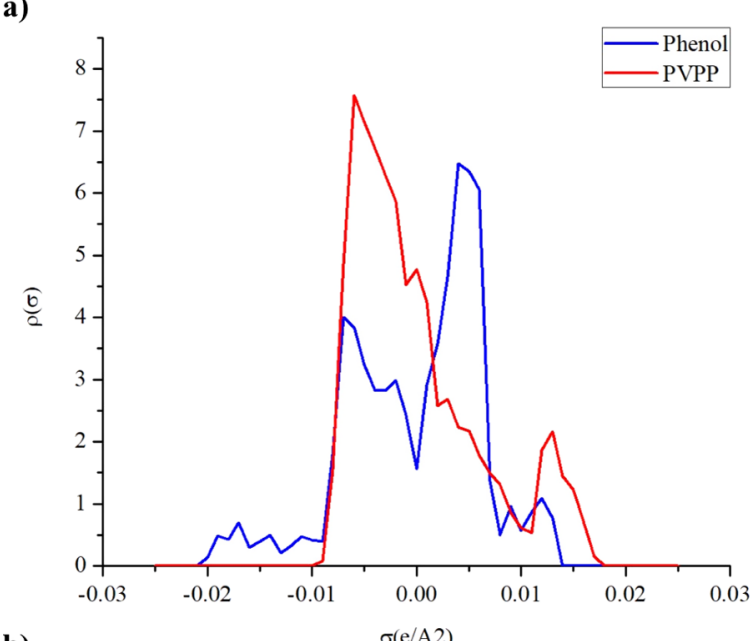

b)

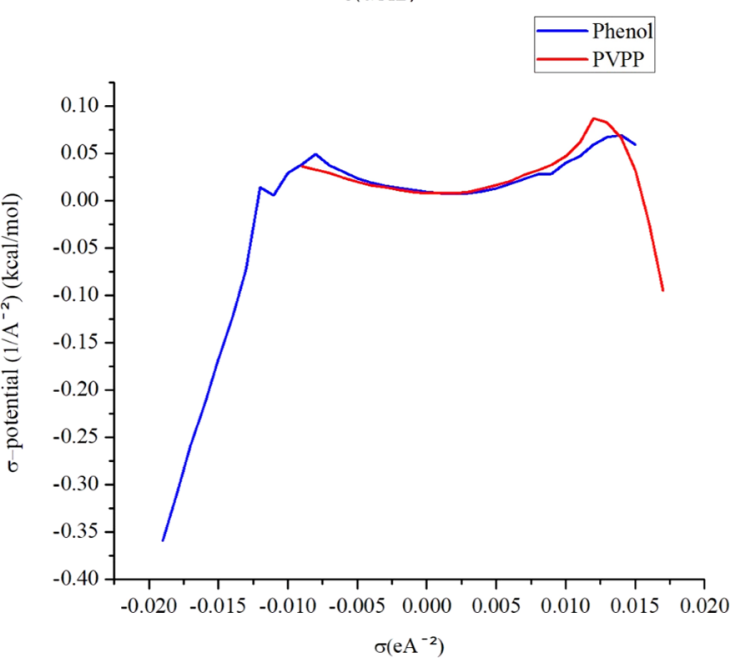

Figure 7. (a) Sigma profile and (b) sigma potential of the PVPP and phenol.

HBA region, while phenol has a peak in the HBD region. These indicate that during the phenol removal, the PVPP and phenol act as an electron donor and electron acceptor pair during the intermolecular hydrogen bond formation, respectively. The peaks of PVPP in the $\sigma$-profile are attributed to the oxygen atom in the carbonyl group, while the peaks in the phenol belong to the hydrogen atom in the hydroxyl group.
Figure $7 \mathrm{~b}$ shows the $\sigma$-potential of the PVPP-phenol complex. Based on the figure, the PVPP and the phenol have a negative $\sigma$-potential in the HBA and HBD regions, respectively. The electron donor, PVPP, has a strong affinity toward the electron acceptor, phenol, which forms the intermolecular hydrogen bond. Both molecules also show repulsive behavior in the hydrophobic region due to the positive $\sigma$-potential in the mixed solution. This result is supported by and aligned with the NCI plot findings, which show that the hydrogen bonding is the main intermolecular interaction that contributed to the removal of the phenol by using PVPP. Meanwhile, hydrophobic interactions only occurred within the PVPP and phenol.

2.2.7. Molecular Electrostatic Potentials (MEPs). The MEP is calculated based on electrostatic potential of the molecule. It is one of the informative tools to predict the reactive site for nucleophilic and electrophilic attack ${ }^{23}$ during the hydrogen bonding interaction between cropovidone and phenol. In this study, MEP surfaces were computed and derived from B3LYP/ $6-311^{++} \mathrm{G}(\mathrm{d}, \mathrm{p})$ in PCM method calculations using Gaussian $09 \mathrm{~W}$ and visualized in Gaussian View 5.0. These surfaces corresponded to an isodensity value of 0.001 a.u. The noncovalent interactions (hydrogen bonds) occurring on the molecular surface of the PVPP-phenol complex are notable.

In Figure S5, the red and blue in color represent the most nucleophilic and electrophilic regions in the PVPP-phenol complex. In the hydrogen bond formation model, the hydrogen bond was formed between atom O9 (PVPP) and atom H13 (phenol). This is because atom $\mathrm{O} 9$ is naturally high in electronegativity (red) compared to $\mathrm{H} 13$, which has low electronegativity (blue). Here, the $\mathrm{H} 13$ atom tended to accept a nucleophile and the $\mathrm{O} 9$ atom tended to receive an electrophile. However, in addition to O9 (PVPP) and H13 (phenol), other parts were also highly nucleophilic and electrophilic. In these cases, the interactions must follow the hard and soft acids and bases (HSAB) principle before the PVPP-phenol complex can form.

2.2.8. Chemical Description. 2.2.8.1. Global Reactivity Descriptors. The HOMO and LUMO of the PVPP-phenol complex is an indicator used to predict the noncovalent interactions that probably occur in the complex. Ideally, the HOMO and LUMO should come from opposite molecules so that the interaction is favorable. Based on Figure 8, we determined that the HOMO and LUMO of the PVPP-phenol

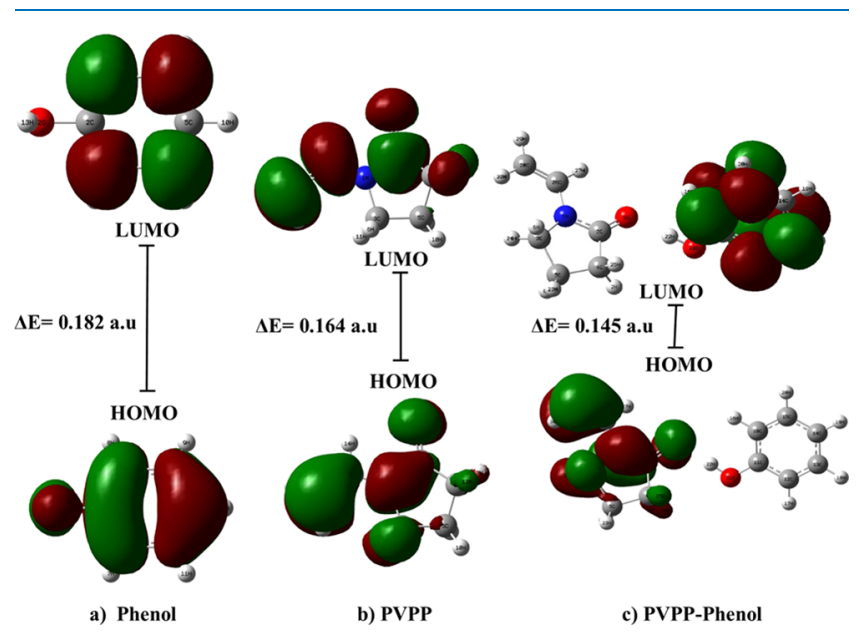

Figure 8. HOMO-LUMO of (a) phenol, (b) PVPP, and (c) PVPPphenol. 
Table 5. Calculation of Quantum Chemical Parameters Based on DFT Methods B3LYP 6-311 ${ }^{++}$G (d,p) of PVPP, Phenol, and PVPP-Phenol Complex Samples

\begin{tabular}{|c|c|c|c|c|c|c|c|c|c|}
\hline structure & E HOMO (a.u.) & E LUMO (a.u.) & $\eta($ a.u. $)$ & $\mu$ (a.u.) & $\omega$ (a.u.) & $\chi($ a.u. $)$ & $S$ (a.u.) & HOMO-LUMO gap (a.u.) & $\Delta N$ \\
\hline PVPP (1) & -0.312 & -0.149 & 0.082 & 0.230 & 0.325 & -0.230 & 6.099 & 0.164 & 1.412 \\
\hline phenol (2) & -0.348 & -0.166 & 0.091 & 0.257 & 0.403 & -0.257 & 5.948 & 0.182 & 1.567 \\
\hline PVPP-phenol complex (3) & -0.330 & -0.185 & 0.073 & 0.258 & 0.458 & -0.258 & 6.849 & 0.145 & 1.767 \\
\hline
\end{tabular}

complex were located mainly on the PVPP and phenol, respectively. The HOMO-LUMO band gap energy is an important parameter for comparing the reactivity of the phenol with PVPP; the lower the band gap energy of a molecule, the more reactive the molecules are and vice versa. ${ }^{51}$ The gap between them is the energy required to excite an electron from the HUMO to the LUMO and is equal to the chemical hardness. The larger the energy gap of a molecule, the lower its polarizability. ${ }^{52}$ Based on Table 5 and Figure 8, PVPP (0.164 a.u.) has a higher reactivity than phenol (0.182 a.u.).

The absolute hardness $(\eta)$ and softness $(S)$ are important parameters in the measurement of a molecule's stability and reactivity. ${ }^{54} \mathrm{~A}$ hard molecule has a large energy gap, whereas a soft molecule has a smaller gap. Soft molecules are more reactive than hard molecules because they can easily donate electrons to an acceptor. As presented in Table 5, phenol (0.091 a.u.) has a higher value of $\eta$ than PVPP (0.082 a.u.) does, whereas PVPP (6.099 a.u.) has a higher $S$ value than phenol (5.948 a.u.). These results are consistent with the band gap energies of PVPP and phenol-molecules that have a lower band-gap energy will be more reactive (i.e., they tend to donate electrons).

Chemical potential energy $(\mu)$ can be defined as the tendency and ability of an atom, molecule, or functional group to attract electrons (electron density) toward itself to react; in other words, the higher the value of $\mu$, the harder it is for the molecule to interact. Electronegativity $(\chi)$ is the chemical potential energy that describes the tendency of an atom to attract electrons to become anionic; in other words, it is a measurement of the reactivity of a component. The higher the value $\chi$ of an atom, the more electrons that are attracted toward it. ${ }^{53}$ The electrophilicity index $(\omega)$ is a measure of the ability of a monomer to be electrophilic. ${ }^{51}$ Based on the values of $\mu, \chi$, and $\omega$ in Table 5, PVPP is more reactive than phenol. This is because the values of $\mu, \chi$, and $\omega$ for PVPP are $0.230,-0.230$, and 0.325 a.u. compared to the values for phenol, which are $0.257,-0.257$, and 0.403 a.u., respectively.

Charge transfer is important in hydrogen bond formation. Therefore, the maximum charge transfer $(\Delta N)$ for PVPP and phenol was measured to determine the selectivity of a monomer to act as an electron donor or electron acceptor. $\Delta N$ was calculated according to eq 4 . The electrophilic charge transfer (ECT) is defined as the difference between $\Delta N_{\max }$ values of interacting molecules. ${ }^{54}$ The ECT for each monomer (PVPP and phenol) was calculated based on eq 5 , and the results were compared to determine the behavior of the monomer, that is, to determine whether it is nucleophilic or electrophilic. The calculated ECT value was less than zero $(-0.155$ a.u.) for reactant molecules PVPP (1) and phenol (2), indicating that charge flows from PVPP (1) to (2). Referring to eqs 6 and 7 below, it can be stated that PVPP undergoes nucleophilic substitution by donating electrons to phenol to form a hydrogen bond $($ ECT value $<0)$.

$$
\Delta N=-\mu^{2} / \eta
$$

$$
\begin{aligned}
& \operatorname{ECT}=\left(\Delta N_{\max (1)}\right)-\left(\Delta N_{\max (2)}\right) \\
& \mu_{1}^{2} / \eta_{1}-\mu_{2}^{2} / \eta_{2}<0 \\
& \mu_{1}^{2} / \eta_{1}-\mu_{2}^{2} / \eta_{2}>0
\end{aligned}
$$

where eq 6 states that the negative charge has transferred from monomer PVPP (1) to phenol (2) and monomer (1) acts as a nucleophilic, and eq 7 states that the negative charge has moved from monomer (2) to (1).

2.2.8.2. Local Reactivity Descriptors. Local reactivity descriptors are important for determining the resulting product. Table 6 shows the quantum chemical parameters for selected

\begin{tabular}{|c|c|c|c|}
\hline function & atom & $f_{\mathrm{k}}^{-}$ & $S f_{\mathrm{k}}^{-}$ \\
\hline \multirow[t]{4}{*}{ electrophilic attack } & PVPP & & \\
\hline & O9 & 0.147 & 0.897 \\
\hline & $\mathrm{C} 15$ & 0.140 & 0.854 \\
\hline & H16 & 0.092 & 0.561 \\
\hline \multirow{4}{*}{ nucleophilic attack } & phenol & & \\
\hline & H13 & 0.407 & 0.164 \\
\hline & $\mathrm{C} 1$ & 0.328 & 0.132 \\
\hline & C3 & 0.326 & 0.131 \\
\hline
\end{tabular}

Table 6. Calculated Quantum Chemical Descriptors for the Electrophilic $\left(f_{\mathrm{k}}^{-1}\right.$ and $\left.S f_{\mathrm{k}}^{-}\right)$and Nucleophilic $\left(f_{\mathrm{k}}^{+}\right.$and $\left.\omega f_{\mathrm{k}}^{+}\right)$ Attacks for the Monomers (Phenols and PVPP)

atoms involved in the formation of the PVPP-phenol complex. The terms $f_{\mathrm{k}}^{+}, \mathrm{Sf}_{\mathrm{k}}^{+}, f_{\mathrm{k}}^{-}$, and $\omega f_{\mathrm{k}}^{-}$are local descriptors that represents the favored regions in which a chemical species will alter its electron density when the number of electrons is changed. Therefore, they indicate the propensity of the electronic density to distort at a certain position upon donating or accepting electrons. ${ }^{55,56}$ As discussed for the global reactivity descriptors, PVPP and phenol are prone to act as a nucleophile and electrophile, respectively. However, the tendency of atoms in PVPP and phenol to undergo nucleophilic and electrophilic molecule adsorption is in the orders $\mathrm{O} 9>\mathrm{C} 15>\mathrm{H} 16$ and $\mathrm{H} 13>$ $\mathrm{C} 1>\mathrm{C} 3$, respectively. The higher the $f_{\mathrm{k}}{ }^{-}$and $\mathrm{Sf}_{\mathrm{k}}{ }^{-}$values, the higher the percentage of atoms that are nucleophilic, and the higher the $f_{\mathrm{k}}{ }^{+}$and $\omega f_{\mathrm{k}}{ }^{+}$values, the higher the percentage of atoms that are electrophilic. According to the Fukui function, the proposed mechanism of phenol removal begins with the formation of the PVPP-phenol complex through hydrogen bonding that occurs between the highest nucleophilicity atom (O9, PVPP) and the highest electrophilicity (H13, phenol) atom. This result is in agreement with the HSAB principle, which explains that hard and soft acids prefer to coordinate with hard and soft bases, respectively. ${ }^{57,58}$

\section{CONCLUSIONS}

In conclusion, at $\mathrm{pH} 6.5$, PVPP was successfully used to remove phenol from an aqueous mixture. The results from the adsorption isotherm indicated that its fit corresponds best to 
Langmuir models and follows second-order reaction kinetics. The interactions formed between PVPP and phenol were successfully experimentally characterized using $1 \mathrm{D}$ and 2D IR spectroscopy by showing a shift in the wavenumbers of the functional group involved in the interaction. These groups were the carboxyl group of PVPP and the hydroxyl group of phenol. The types of interactions that contributed to the adsorption efficiency of PVPP in phenol removal were successfully investigated and identified. A theoretical calculation found that the chemical reactivity and stability of the complex are temperature-dependent, as calculated by the DFT approach. As shown by QTAIM, RDG-NCI, and COSMO-RS analyses, removal of phenol by PVPP occurred due to the hydrogen bonding interaction that formed in the complex, which was successfully identified as an intermediate type of hydrogen bonding $(\nabla 2 \rho(r)>0$ and $H<0)$. The adsorption mechanism that occurs in the system is due to PVPP acting as a nucleophile, whereas phenol acts as an electrophile. During hydrogen bond formation, the $\mathrm{O} 9$ atom (donor electron) reacts with the $\mathrm{H} 22$ atom (acceptor electron), as calculated based on its global and local reactivity descriptors. All experimental and theoretical results were in good agreement in terms of the types of interactions involved in the system and confirmed that the adsorption efficiency of PVPP in phenol removal was up to $74.91 \%$.

\section{EXPERIMENTAL AND THEORETICAL SECTION}

4.1. Reagents and Chemicals. PVPP $\left(\mathrm{C}_{6} \mathrm{H}_{9} \mathrm{NO}\right)_{n}$, FolinCiocâlteu (Folin reagent), sodium carbonate $\left(\mathrm{Na}_{2} \mathrm{CO}_{3}\right)$, gallic acid $\left(\mathrm{C}_{6} \mathrm{H}_{2}(\mathrm{OH})_{3} \mathrm{COOH}\right)$, citric acid-1-hydrate $((\mathrm{COOH})$ $\mathrm{CH}_{2} \mathrm{COOH} \cdot \mathrm{H}_{2} \mathrm{O}$ or $\left.\mathrm{C}_{6} \mathrm{H}_{10} \mathrm{O}_{8}\right)$, monobasic sodium phosphate $\left(\mathrm{NaH}_{2} \mathrm{PO}_{4}\right)$, hydrochloric acid $(\mathrm{HCl})$, and phenol $\left(\mathrm{C}_{6} \mathrm{H}_{5} \mathrm{OH}\right)$ were bought from Sigma, Malaysia.

4.2. Purification of PVPP. The use of purified PVPP is essential in removing phenol from an aqueous medium. PVPP may contain traces of metal ions and vinylpyrrolidone monomer as it is commonly produced in beverage processing. PVPP was purified by boiling it in $10 \%(\mathrm{v} / \mathrm{v}) \mathrm{HCl}$ for $10 \mathrm{~min}$ and then washing with glass-distilled water until free of chloride ions. The purified PVPP then was dried and stored. ${ }^{16}$

4.3. Total Phenolic Content. The total phenolic content was measured using the Folin-Ciocâlteu assay with some minor modifications. ${ }^{59}$ Briefly, $1 \mathrm{~mL}$ of treated sample was added to 0.5 $\mathrm{mL}$ of Folin reagent; after $3 \mathrm{~min}, 0.5 \mathrm{~mL}$ of $1 \mathrm{M} \mathrm{Na}_{2} \mathrm{CO}_{3}$ was added. The tube was shaken and left to stand for $1 \mathrm{~h}$, after which absorbance was measured at $725 \mathrm{~nm}$. The concentration of total phenol in the sample was determined from a gallic acid standard graph and expressed as micrograms of gallic acid. Total phenolic content was further used in eq 8 to calculate the percentage of phenol removal from the aqueous medium

$$
\operatorname{removal}(\%)=\frac{C_{\mathrm{o}}-C_{\mathrm{e}}}{C_{\mathrm{o}}} \times 100
$$

where $C_{\mathrm{o}}$ and $C_{\mathrm{e}}$ are the initial and equilibrium $(\mathrm{mg} / \mathrm{ml})$ of phenol, respectively.

4.4. Adsorption Assay. The adsorption of phenol by PVPP was conducted according to the methods reported by refs 60 and 61 with minor modifications. The phenol was prepared at a concentration of $0.5 \mathrm{mg} / \mathrm{mL}$ in $10 \mathrm{~mL}$ of two buffer solutions: a $50 \mathrm{mM}$ citrate-phosphate buffer with $\mathrm{pH} 3.5$ and a $50 \mathrm{mM}$ phosphate buffer with $\mathrm{pH}$ 6.5. Phenol was continuously stirred and heated at a moderate temperature until the mixture was homogeneous. The interaction of PVPP and phenol was evaluated by adding $0.25 \mathrm{mg}$ of PVPP to the sample mixtures. The ratio by weight of phenol to PVPP was $2: 1 \mathrm{w} / \mathrm{w}$. The samples were incubated at $25{ }^{\circ} \mathrm{C}$ with stirring at a certain time range $(0-120 \mathrm{~min}$, with a $10 \mathrm{~min}$ interval) to allow binding of the phenol to the PVPP; the mixtures were then centrifuged at $4500 \mathrm{rpm}$ (HermLe Z 323K, Germany) for $25 \mathrm{~min}$ at $25^{\circ} \mathrm{C}$. The treated samples (those with added PVPP) were passed through filter paper (Whatman, no. 54) to remove the PVPP-phenol complexes. The supernatant and precipitate were then collected and stored at $4{ }^{\circ} \mathrm{C}$ until further analysis.

4.4.1. Adsorption Kinetic Analysis. The kinetics studies were carried out with $2.5 \mathrm{mg}$ of adsorbent in phenol solution with an initial concentration of $0.50 \mathrm{mg} / \mathrm{mL}$ at $\mathrm{pH} 6.5$ and $\mathrm{pH} 3.5$ and at $25^{\circ} \mathrm{C} .^{60}$ The phenol concentration was measured at $10 \mathrm{~min}$ set time intervals until $120 \mathrm{~min}$ by spectrophotometry at $725 \mathrm{~nm}$. The adsorption capacity at time $t(q t)$ was calculated by eq 9:

$$
q t=\frac{\left(C_{\mathrm{o}}-C_{\mathrm{t}}\right) V}{m}
$$

where $C_{\mathrm{o}}$ is the phenol initial concentration in the liquid phase $\left(\mathrm{mg} \mathrm{ml}^{-1}\right), C_{\mathrm{t}}$ is the phenol concentration in the liquid phase at time $t\left(\mathrm{mg} \mathrm{ml}^{-1}\right), m$ is the amount of adsorbent $(\mathrm{mg})$, and $V$ is the volume of the solution $(\mathrm{mL})$.

In order to test the validation of data, PFO and PSO models were applied using the following equations, eqs 10 and 11 , respectively:

$$
\log \left(q_{\mathrm{e}}-q_{\mathrm{t}}\right)=\log q_{\mathrm{e}}-\frac{k}{2.303} t
$$

where $q_{\mathrm{e}}$ is the quantity of phenol uptake at equilibrium $(\mathrm{mg} / \mathrm{g})$, $q_{\mathrm{t}}$ is the quantity of phenol uptake at time $t(\mathrm{mg} / \mathrm{g}), k_{1}$ is the rate constant $\left(\min ^{-1}\right)$ for the plot of $\log \left(q_{\mathrm{e}}-q_{\mathrm{t}}\right)$ against $t$. By plotting the $\log \left(q_{\mathrm{e}}-q_{\mathrm{t}}\right)$ against $t$, a straight line was obtained. The slope and intercept values were used to calculate $\log q_{\mathrm{e}}$ and $k_{1}$ for the adsorption of phenol, respectively:

$$
\frac{t}{q t}=\frac{1}{k_{2} q_{\mathrm{e}}^{2}}+\frac{1}{q_{\mathrm{e}}} t
$$

where $k_{2}(\mathrm{~g} / \mathrm{mg} \min )$ is the second-order rate constant and slope $\left(1 / q_{\mathrm{e}}\right)$ and intercept $\left(1 / k_{2} q_{\mathrm{e}}^{2}\right)$ were determined from the linear plot of $t / q t$ versus $t$.

4.4.2. Adsorption Isotherms. The adsorption equilibrium of phenol onto PVPP was elucidated by using the batch-adsorption method. This method was carried out by changing the initial concentration of phenol ions from 0.5 to $2.5 \mathrm{mg} / \mathrm{mL}$ using 25 $\mathrm{mg}$ of PVPP of phenol solution at $\mathrm{pH} 6.5$ with a 90 min contact time at $200 \mathrm{rpm}$. The effect of the phenol ion concentration on PVPP was also examined using eqs 12 and $13 .{ }^{61,62}$ The separation factor, which is important for the Langmuir isotherm characteristic, is determined using eq 14

$$
\begin{aligned}
& \text { Langmuir: } q_{e}=\frac{q_{\max } K_{\mathrm{L}} C_{\mathrm{e}}}{1+K_{\mathrm{L}} C_{\mathrm{e}}} \\
& \text { Freundlich: } \log q_{\mathrm{e}}=\log K_{\mathrm{f}}+\frac{1}{n} \log C_{\mathrm{e}}
\end{aligned}
$$

where $q_{\mathrm{e}}$ is the quantity of phenol ions captured by unit mass of PVPP, $C_{\mathrm{e}}$ is the amount of phenol ions in the residual solution at equilibrium, $K_{\mathrm{L}}$ is the Langmuir constant, and $K_{\mathrm{f}}$ is Freundlich constant. 


$$
R_{\mathrm{L}}=\frac{1}{\left(1+K_{\mathrm{L}} C_{\mathrm{i}}\right)}
$$

where $C_{\mathrm{i}}$ is the concentration of phenol ions before adsorption. The parameter $R_{\mathrm{L}}$ indicates the shape of the isotherm accordingly: $R_{\mathrm{L}}>1$, unfavorable; $R_{\mathrm{L}}=1$, linear; $1>R_{\mathrm{L}}>0$, favorable; $R_{\mathrm{L}}=0$, irreversible.

4.5. Statistical Analysis. All phenolic content data, both with treatment (PVPP) and without treatment (control), were obtained in triplicate and expressed as mean \pm standard deviation (SD). Comparison and statistical significance $(p<$ 0.05 ) of mean between the control and treatment data were performed using one-way analysis of variance (ANOVA) and Tukey's test, respectively. All statistical analysis was done using JMP Pro 13 (SAS Institute Inc., USA).

4.6. 1D and 2D IR Analysis. FTIR analysis was carried out using a PerkinElmer FTIR equipped with $2 \mathrm{D}$ IR using $\mathrm{KBr}$ pellets. This analysis was conducted to determine the functional groups of compounds present in the mixture aqueous medium. The spectra of 1D spectroscopy were recorded from 4000 to 600 $\mathrm{cm}^{1}$. The 2D IR spectra were recorded from 50 to $120^{\circ} \mathrm{C}$ at 10 ${ }^{\circ} \mathrm{C}$ intervals with correlation analysis software created by Tsinghua University, Beijing, China. ${ }^{63}$

4.7. QTAIM Analysis. The electronic structure of the PVPP-phenol complex was further explored using QTAIM methods using the B3LYP/6-31 $1^{++} \mathrm{G}(\mathrm{d}, \mathrm{p})$ basis to obtain deeper insights into the hydrogen bond interactions. The QTAIM analysis was performed using AIMAII professional software (version 19.10.12). ${ }^{46,47}$

4.8. RDG-NCI Analysis. The optimized structure of the PVPP-phenol complex was analyzed by RDG-NCI with NCIPlot 3.0, and the isosurfaces were graphed with Visual Molecular Dynamics. The graph enables the $2 \mathrm{D}$ visualization of the noncovalent interaction peaks in the reduced density gradient (RDG) at low densities. 14,64

4.9. Conductor-like Screening Model for Real Solvents (COSMO-RS) Calculation. The PVPP $\left(\mathrm{C}_{6} \mathrm{H}_{9} \mathrm{NO}\right)_{n}$ and phenol $\left(\mathrm{C}_{6} \mathrm{H}_{5} \mathrm{OH}\right)$ structures were taken from the PubChem database (CID: 996 and 6917). In this study, the Becke-Perdew-86 (BP86) functional and the triple-zeta valence potential basis set were employed to optimize and calculate the continuum solvation COSMO of the PVPP and phenol structures. Next, the COSMO file, which contains the screening charge density on the surface of each molecule, was generated. All quantum chemical calculations were carried out using the Amsterdam Density Functional package. ${ }^{65}$

4.10. DFT Calculation. DFT is an effective theoretical method that can be used to determine the interactions between PVPP and phenol. However, because of the computational cost of optimizing the PVPP and phenol structures, the current study focused on the monomer of PVPP ( $N$-vinylpyrrolidone) ${ }^{66,67}$ and phenol. ${ }^{54,68}$ Three-dimensional structures of PVPP and phenol were taken from the PubChem database. The PES of the PVPP-phenol complex was obtained either by keeping all the atoms fixed except for the proton in the hydrogen bridge or in a nonadiabatic way where the structure was optimized for particular $\mathrm{OH}$ distances that were kept constant. For the intermolecular hydrogen bonds, the outcome of the two methods was the same. These results show that a nonadiabatic PES was produced for $\mathrm{OH}(\mathrm{O} 9-\mathrm{H} 22-\mathrm{O} 21)$ distances that changed from 1.5 to $5.1 \AA$ with $0.1 \AA$ steps, using the semiempirical PM6 method in the Gaussian suite 09.
All the investigated 3D structures (PVPP, phenol, and PVPPphenol complex) were fully optimized using DFT B3LYP 6$311^{++} \mathrm{G}(\mathrm{d}, \mathrm{p})$ in the PCM using the Gaussian 09 suite of programs due to the efficiencies of these programs. ${ }^{69}$ B3LYP was used to determine the energy of intermolecular hydrogen bonding interactions $\left(E_{\text {int }}\right)$. B3LYP is a hybrid functional, a combination of the gradient functional proposed by Beckee ${ }^{70}$ and the Hartree-Fock local exchange function ${ }^{71}$ was used to determine the energy of intermolecular hydrogen bonding interactions $\left(E_{\text {int }}\right)$. The $E_{\text {int }}$ that formed during PVPP-phenol complex formation was calculated according to eq 15 and by defining the energy differences between the complex, the isolated monomers, ${ }^{66}$ and the basis set superposition errors (BSSEs)

$$
\left(E_{\text {int }}\right)=E_{(\text {PVPP-phenol })}-\left[E_{(\mathrm{PVPP})}+E_{(\text {phenol })}\right]-\text { BSSE }
$$

where $E_{(\mathrm{PVPP}-\text { phenol }}$ is the energy of PVPP-phenol complex formation, $E_{(\mathrm{PVPP})}$ is the energy of PVPP formation, and $E_{\text {(phenol) }}$ is the energy of phenol formation.

The structural properties of charge distribution, $\mathrm{HOMO}$ energies $\left(E_{\mathrm{HOMO}}\right)$, LUMO energies $\left(E_{\mathrm{LUMO}}\right)$, bond length, electron affinities, quantum chemical descriptors of the electron affinity, hardness, ionization potential, electrophilicity, chemical potential, local softness, thermodynamic parameters, MEP, and Fukui function were calculated directly from the orbital energies of the optimized geometries. ${ }^{72,73}$ In addition, the electronic transition for PVPP-phenol was calculated based on TD-DFT using the B3LYP/6-311 ${ }^{++} \mathrm{G}(\mathrm{d}, \mathrm{p})$ basis sets. All the above calculations were carried out using Gaussian09 software.

\section{ASSOCIATED CONTENT}

\section{Supporting Information}

The Supporting Information is available free of charge at https://pubs.acs.org/doi/10.1021/acsomega.1c02699.

Supplementary figure details regarding the potential energy curve (PES) for the crospovidone-phenol complex, optimal structures and Mulliken atomic charge using DFT B3LYP 6-311G ++ (d,p), QTAIM, and MEP and supplementary data details regarding the selected bond lengths and bond angles for the formation of hydrogen bond, data of energy of hydrogen bond interaction, calculated thermodynamic parameters, and thermodynamic properties at different temperatures (PDF)

\section{AUTHOR INFORMATION}

\section{Corresponding Author}

Mohammad Norazmi Ahmad - Experimental and Theoretical Research Lab, Department of Chemistry, Kulliyyah of Science, International Islamic University of Malaysia, 25200 Kuantan, Pahang, Malaysia; River of Life (ROL) Kuantan Chapter, International Islamic University of Malaysia, 25200 Kuantan, Pahang, Malaysia; Innovative Toyyib Environment Minds (ITEMS), International Islamic University of Malaysia, 25200 Kuantan, Pahang, Malaysia; Drug and Poison Call Centre, IIUM Poison Centre, International Islamic University of Malaysia, 25200 Kuantan, Pahang, Malaysia; ○ orcid.org/0000-0001-5742-0346; Email: mnorazmi85@ gmail.com, mnorazmi@iium.edu.my 


\section{Authors}

Muhammad Ammar Mohammad Alwi - Experimental and Theoretical Research Lab, Department of Chemistry, Kulliyyah of Science, International Islamic University of Malaysia, 25200 Kuantan, Pahang, Malaysia

Erna Normaya - Experimental and Theoretical Research Lab, Department of Chemistry, Kulliyyah of Science, International Islamic University of Malaysia, 25200 Kuantan, Pahang, Malaysia; River of Life (ROL) Kuantan Chapter, International Islamic University of Malaysia, 25200 Kuantan, Pahang, Malaysia; Innovative Toyyib Environment Minds (ITEMS), International Islamic University of Malaysia, 25200 Kuantan, Pahang, Malaysia

Hakimah Ismail - Experimental and Theoretical Research Lab, Department of Chemistry, Kulliyyah of Science, International Islamic University of Malaysia, 25200 Kuantan, Pahang, Malaysia

Anwar Iqbal - School of Chemical Science, Universiti Sains Malaysia, 11800 Penang, Malaysia

Bijarimi Mat Piah - Faculty of Chemical \& Natural Resources Engineering, Universiti Malaysia Pahang, 26300 Kuantan, Pahang, Malaysia

Mohd Armi Abu Samah - Experimental and Theoretical Research Lab, Department of Chemistry, Kulliyyah of Science, International Islamic University of Malaysia, 25200 Kuantan, Pahang, Malaysia; River of Life (ROL) Kuantan Chapter, International Islamic University of Malaysia, 25200 Kuantan, Pahang, Malaysia; Innovative Toyyib Environment Minds (ITEMS), International Islamic University of Malaysia, 25200 Kuantan, Pahang, Malaysia

Complete contact information is available at:

https://pubs.acs.org/10.1021/acsomega.1c02699

\section{Notes}

The authors declare no competing financial interest.

\section{ACKNOWLEDGMENTS}

This paper is a part of a project that was funded by the Malaysia Ministry of Higher Education (FRGS19/1/2019/STG01/ UIAM/02/2) and International Islamic University Malaysia (Grant No. IRF 19-014-0014).

\section{REFERENCES}

(1) Anku, W. W.; Mamo, M. A.; Govender, P. P. Phenolic compounds in water: Sources, reactivity, toxicity, and treatment methods, in: SotoHernandez, M., Palma-Tenango, M., Garcia-Mateos, R., Phenolic compounds: Natural sources, importance and applications; IntechOpen Publ. 2017, p 419-443.

(2) Trubetskaya, A.; Kling, J.; Ershag, O.; Attard, T.; Schröder, E. Removal of phenol and chlorine from wastewater using steam activated biomass soot and tire carbon black. J. Hazard. Mater. 2019, 365, 846856.

(3) Zhu, Y.; Liang, J.; Yang, Q.; Zhou, H.; Peng, K. Water use of a biomass direct-combustion power generation system in China: A combination of life cycle assessment and water footprint analysis. Renew. Sustain. Energy. Rev. 2019, 115, 109396.

(4) Sun, H. L.; Chuai, J.; Wei, H.; Zhang, X.; Yu, H. Multi-functional organic gelator derived from phenyllactic acid for phenol removal and oil recovery. J. Hazard. Mater. 2019, 366, 46-53.

(5) Singh, S.; Mishra, R.; Sharma, R. S.; Mishra, V. Phenol remediation by peroxidase from an invasive mesquite: Turning an environmental wound into wisdom. J. Hazard. Mater. 2017, 334, 201-211.

(6) Salari, M.; Dehghani, M. H.; Azari, A.; Motevalli, M. D.; Shabanloo, A.; Ali, I. High performance removal of phenol from aqueous solution by magnetic chitosan based on response surface methodology and genetic algorithm. J. Hazard. Mater. 2019, 2851, 146-157.

(7) Zhang, M.; Zhang, Z.; Liu, S.; Peng, Y.; Chen, J.; Ki, S. Y. Ultrasound-assisted electrochemical treatment for phenolic wastewater. Ultrason. Sonochem. 2020, 65, 105058.

(8) Tyagi, M.; Kumari, N.; Jagadevan, S. A holistic Fenton oxidationbiodegradation system for treatment of phenol from coke oven wastewater: Optimization, toxicity analysis and phylogenetic analysis. J. Water Process Eng. 2020, 37, 101475.

(9) Kajani, A. A.; Bordbar, A.-K. Biogenic magnetite nanoparticles: A potent and environmentally benign agent for efficient removal of azo dyes and phenolic contaminants from water. J. Hazard. Mater. 2019, 366, 268-274.

(10) Castro, R. I.; Forero-Doria, O.; Guzmán, L.; Lauriea, V. F.; Valdes, O.; Ávila-Salasa, F.; López-Cortésa, X.; Santosa, L. S. New polymer for removal of wine phenolics: Poly $(\mathrm{N}-(3-(\mathrm{N}-$ isobutyrylisobutyramido)-3-oxopropyl)acrylamide) (P-NIOA). Food Chem. 2016, 213, 554-560.

(11) Duran-Lara, E. F.; López-Cortés, X. A.; Castro, R. I.; Avila-Salas, F.; González-Nilo, F. D.; Laurie, V. F.; Santos, L. S. Experimental and theoretical binding affinity between polyvinylpolypyrrolidone and selected phenolic compounds from food matrices. Food Chem. 2015, 168, 464-470.

(12) Li, D.; Yang, X.; Zhou, Z.; Jiang, B.; Tawfik, A.; Zhao, S.; Men, F. Molecular traits of phenolic moieties in dissolved organic matter: Linkages with membrane fouling development. Environ. Int. 2019, 133, 105202.

(13) Folch-Cano, C.; Olea-Azar, C.; Speisky, H. Structural and thermodynamic factors on the adsorption process of phenolic compounds onto polyvinylpolypyrrolidone. Colloids Surf. A Physicochem. Eng. Asp. 2013, 418, 105-111.

(14) Normaya, E.; Hamdan, M. F. A.; Ahmad, M. N.; Aziz, Y. F. A.; Bulat, K. H. K. DFT/TD-DFT study on development and optimization of 1- anilino-3- phenyliminourea as a colorimetric chemosensor for $\mathrm{Hg}^{2+}$ recognition in aqueous medium. J. Mol. Struct. 2020, 1206, $1-7$.

(15) Bai, X. M.; Ni, F. Y.; Tang, S.; Zhao, Y. Z.; Pan, Z. DFT analysis on the removal of dimethylbenzoquinones in atmosphere and water environments: $\cdot \mathrm{OH}$-initiated oxidation and captured by $(\mathrm{TiO} 2) \mathrm{n}$ clusters ( $\mathrm{n}=1-6)$. J. Hazard. Mater. 2020, 386, 121636.

(16) Gil, M.; Avila-Salas, F.; Santos, L. S.; Iturmendi, N.; Moine, V.; Cheynier, V.; Saucier, C. Rosé wine fining using polyvinylpolypyrrolidone: Colorimetry, targeted polyphenomics, and molecular dynamics simulations. J. Agric. Food Chem. 2017, 65, 10591-10597.

(17) Wei, D.; Zhao, C.; Khan, A.; Sun, L.; Ji, Y.; Ai, Y.; Wang, X. Sorption mechanism and dynamic behavior of graphene oxide as an effective adsorbent for the removal of chlorophenol based environmental-hormones: A DFT and MD simulation study. Chem. Eng. J. 2019, 375, 121964

(18) Abdelwahab, O.; Amin, N. K. Adsorption of phenol from aqueous solutions by Luffa cylindrica fibers: Kinetics, isotherm and thermodynamic studies. Egypt. J. Aquat. Res. 2013, 39, 215-223.

(19) Mojoudi, N.; Mirghaffari, N.; Soleimani, M.; Shariatmadari, H.; Belver, C.; Bedia, J. Phenol adsorption on high microporous activated carbons prepared from oily sludge: equilibrium, kinetic and thermodynamic studies. Sci. Rep. 2019, 9, 19352.

(20) Issabayeva, G.; Hang, S. Y.; Wong, M. C.; Aroua, M. K. A review on the adsorption of phenols from wastewater onto diverse groups of adsorbents. Rev. Chem. Eng. 2018, 34, 855-873.

(21) Villegas, L. G. C.; Mashhadi, N.; Chen, M.; Mukherjee, D.; Taylor, K. E.; Biswas, N. A Short Review of Techniques for Phenol Removal from Wastewater. Curr. Pollut. Rep. 2016, 2, 157-167.

(22) He, Y.; Liu, F.; Bian, W.; Liu, X.; Ma, S.; Zheng, X.; Du, Z.; Zhang, K.; Ouyang, D. Molecular interactions for the curcuminpolymer complex with enhanced anti-inflammatory effects. Pharmaceutics. 2019, 11, 442.

(23) Pavia, L.; Lampman, G. M.; Kriz, G. S.; Vyvyan, J. R. Introduction to Spectroscopy; Fifth ed, Cengage Learning: Stamford, United States of America, 2015, 14-72. 
(24) Popescu, M. C.; Froidevaux, J.; Navi, P.; Popescu, C. M. Structural modifications of Tilia cordata wood during heat treatment investigated by FT-IR and 2D IR correlation spectroscopy. J. Mol. Struct. 2013, 1033, 176-186.

(25) Sun, B.; Lin, Y.; Wu, P.; Siesler, H. W. A FTIR and 2D-IR Spectroscopic study on the microdynamics phase separation mechanism of the poly ( $\mathrm{N}$-isopropylacrylamide) aqueous solution. Macromolecules 2008, 41, 1512-1520.

(26) Noda, I. Generalized two-dimensional correlation method applicable to infrared, Raman, and other types of spectroscopy. Appl. Spectrosc. 1993, 47, 1329-1336.

(27) Noda, I.; Ozaki, Y. Two-dimensional correlation spectroscopy. Applications in Vibrational and Optical Spectroscopy; first ed, John Wiley \& Sons Ltd: Chichester, United Kindgom, 2004, 15-38.

(28) Liu, Y.; Zhang, G. J.; Sun, S. Q.; Noda, I. Study on similar traditional Chinese medicines Cornu Cervi Pantotrichum, Cornu Cervi and Cornu Cervi Degelatinatum by FT-IR and 2D-IR correlation spectroscopy. J. Pharm. Biomed. Anal. 2010, 52, 631-635.

(29) Noda, I. Two-dimensional infrared (2D IR) spectroscopy: Theory and applications. Appl. Spectrosc. 1990, 44, 550-561.

(30) Majerzw, I.; Olovsson, I. The shape of the potential energy curves for NHN+ hydrogen bonds and the influence of non-linearity. Phys. Chem. Chem. Phys. 2008, 10, 3043-3051.

(31) Scott, J. N.; Vanderkooi, J. M. A new hydrogen bond angle/ distance potential energy surface of the quantum water dimer. Water. 2019, 11, 14-28.

(32) Rösel, S.; Becker, J.; Allen, W. D.; Schreiner, P. R. Probing the delicate balance between Pauli repulsion and London dispersion with triphenymethyl derivatives. J. Am. Chem. 2018, 140, 14421-14432.

(33) McDowell, S. A. Cooperativity and bond breakage in model $\mathrm{X}--\mathrm{H}_{3} \mathrm{P}-\mathrm{YZ}\left(\mathrm{X}=\mathrm{F}, \mathrm{Cl} ; \mathrm{YZ}=\mathrm{HF}, \mathrm{ClF}, \mathrm{LiF}, \mathrm{BeH}_{2}\right)$ complexes. Chem. Phys. Lett. 2016, 658, 12-19.

(34) Klöffel, T.; Kozlowska, M.; Popiel, S.; Meye, B.; Rodziewicz, P. Adsorption of sulfur mustard on clean and water saturated $\mathrm{ZnO}(1010)$ : Structural diversity from first principles calculations. J. Hazard. Mater. 2021, 402, 123503.

(35) Al-Ahmary, K. M.; Alenezi, M. S.; Habeeb, M. M. Synthesis, spectroscopic and DFT theoretical studies on the hydrogen bonded charge transfer complex of 4-aminoquinoline with chloranilic acid. $J$. Mol. Liq. 2018, 220, 166-182.

(36) Paul, B. K.; Mahanta, S.; Singh, R. B.; Guchhait, N. A DFT based theoretical study on the photophysics of 4-hydroxyacridine: Single water mediated excited state proton transfer. J. Phys. Chem. A 2010, 114, 2618-2627.

(37) Jia, S.; Yang, Z.; Ren, K.; Tian, Z.; Dong, C.; Ma, R.; Yu, G.; Yang, $\mathrm{W}$. Removal of antibiotics from water in the coexistence of suspended particles and natural organic matters using amino-acid-modifiedchitosan flocculants: A combined experimental and theoretical study. J. Hazard. Mater. 2016, 317, 593-601.

(38) Sun, X. D.; Shi, C. L.; Li, S. H.; Wang, Y. Z.; Qi, D. W.; Wang, W. M.; Yao, H. M.; Jian, Y. W.; Zhu, J. H. New shape-selectivity discovered on graphene-based materials in catching tobacco specific nitrosamines. J. Hazard. Mater. 2018, 358, 234-242.

(39) Herschlag, D.; Pinney, M. M. Hydrogen bonds: simple after all. Biochemistry 2018, 57, 3338-3352.

(40) Balachandran, V.; Karpagam, V.; Revathi, B.; Kavimani, M.; Santhi, G. Conformational stability, vibrational and NMR analysis, chemical potential and thermodynamical parameter of 3-tert-butyl-4hydroxyanisole, Spectrochim. Acta Part A: Mol. Biomol. Spectrosc. 2015, 135, 1039-1051.

(41) Jasmine, N. J.; Arunagiri, C.; Subashini, A.; Stanley, N.; Muthiah, P. T. Synthesis, X-ray structure analysis, thermodynamic and electronic properties of 4-acetamido Benzaldehyde using vibrational spectroscopy and DFT calculations. J. Mol. Struct. 2017, 1130, 244-250.

(42) Uppal, A.; Kour, P.; Kumar, A.; Khajuria, Y. Synthesis, structural, vibrational, electronic, thermal and Fukui analysis of diethyl (hydroxy(4-methoxyphenyl) methyl) phosphonate. J. Mol. Struct. 2018, 1166, 218-227.
(43) Normaya, E.; Baharu, N. A.; Ahmad, M. N. Synthesis of thiosemicarbazone-based colorimetric chemosensor for $\mathrm{Cu} 2+$ ions' recognition in aqueous medium: Experimental and theoretical studies. J. Mol. Struct. 2020, 1212.

(44) Dash, B.; Rath, S. S. Density functional theory and molecular dynamics insights into the site-dependent adsorption of hydrogen fluoride on kaolinite. J. Mol. Liq. 2020, 299, 112265.

(45) Tang, Z.; Wei, H.; Zhou, P. Effects of solvents on the excited state intramolecular proton transfer and hydrogen bond mechanisms of alizarin and its isomers. J. Mol. Liq. 2020, 301, 112415.

(46) Suma, N.; Aruldhas, D.; Joe, I. H.; Anuf, A. R.; Sasi, B. A. Spectroscopic, quantum chemical, QTAIM analysis, molecular dynamics simulation, docking studies and solvent effect of pyridin-2-yl oxyacetic acid herbicide and its derivatives. J. Mol. Struct. 2020, 1206, 127677.

(47) Shukla, S.; Srivastava, A.; Kumar, P.; Tandon, P.; Maurya, R.; Singh, R. B. Vibrational spectroscopic, NBO, AIM, and multiwfn study of tectorigenin: A DFT approach. J. Mol. Struct. 2020, 1217, 128443.

(48) Hossain, R.; Hasan, M.; Nishat, M.; Ahmed, F.; Ferdous, T.; Hossain, M. A. DFT and QTAIM investigations of the adsorption of chlormethine anticancer drug on the exterior surface of pristine and transition metal functionalized boron nitride fullerene. J. Mol. Liq. 2021, 114627

(49) Tang, W.; Row, K. H. Design and evaluation of polarity controlled and recyclable deep eutectic solvent based biphasic system for the polarity driven extraction and separation of compounds. $J$. Cleaner Prod. 2020, 268, 122306.

(50) Cheng, H.; Li, J.; Wang, J.; Chen, L.; Qi, Z. Enhanced vitamin E extraction selectivity from deodorizer distillate by a biphasic system: A COSMO-RS and experimental study. ACS Sustainable Chem. Eng. 2018, 6, 5547-5554.

(51) Normaya, E.; Ahmad, M. N.; Aziz, Y. F. A.; Bulat, K. H. K. Synthesis, characterization and preliminary study on acetylpyrazine $\mathrm{N}(4)$ butylthiosemicarbazone as a potential CDK2 inhibitor combined with DFT calculations. J. Braz. Chem. Soc. 2018, 29, 2197-2206.

(52) Karmakar, A.; Bandyopadhyay, P.; Benerjee, S.; Mandal, N. C.; Singh, B. Synthesis, spectroscopic, theoretical and antimicrobial studies on molecular charge-transfer complex of 4-(2-thiazolylazo)resorcinol (TAR) with 3,5-dinitrosalicyclic, picric acid, and chloranilic acid. J. Mol. Liq. 2020, 299, 112217.

(53) Nyangiwe, N. N.; Ouma, C. N. M. Adsorption and coadsorption of single and multiple natural organic matter on $\mathrm{Ag}$ (111) surface: A DFT study. Appl. Surf. Sci. 2020, 505, 144609.

(54) Liu, M.; Chen, J.; Chen, Y.; Zhu, Y. Interaction between smithsonite and carboxyl collectors with different molecular structure in the presence of water: A theoretical and experimental study. Appl. Surf. Sci. 2020, 510, 145410.

(55) Shang, Z.; Zhang, X. Theoretical study on the interactions between silica and the products of 3-mercaptopropylitriethoxysilane (MPTS) with different hydrolysis degrees. Appl. Surf. Sci. 2020, 502, 143853.

(56) Yan, Z.; Zuo, Z.; Li, Z.; Zhang, J. A cluster DFT study of NH3 and NO adsorption on the (MoO2)2+/HZSM-5 surface: Lewis versus Brønsted acid sites. Appl. Surf. Sci. 2014, 321, 339-347.

(57) Xu, L.; Zhang, A.; Zhang, F.; Liu, J. Preparation and characterization of a novel macroporous silica-bipyridine asymmetric multidentate functional adsorbent and its application for heavy metal palladium removal. J. Hazard. Mater. 2017, 337, 178-188.

(58) Dostanić, J.; Lončarević, D.; Zlatar, M.; Vlahović, F.; Jovanović, D. M. Quantitative structure-activity relationship analysis of substituted arylazo pyridine dyes in photocatalytic system: Experimental and theoretical study. J. Hazard. Mater. 2016, 316, 26-33.

(59) Klungsupya, P.; Suthepakul, N.; Muangman, T.; Rerk-am, U.; Thongdon, J. Determinetaion of free radical scavenging, antioxidative DNA damage activities and phytochemical components of active fractions from Lansium domesticum Corr. Fruit. Nutr. 2015, 7, 68526873. 
(60) Alves, D. C. S.; Coseglio, B. B.; Pinto, L. A. A.; Cadaval, T. R. S., Jr. Development of Spirulina/chitosan foam adsorbent for phenol adsorption. J. Mol. Liq. 2020, 309, 113256.

(61) Zagklis, D. P.; Vavouraki, A. I.; Kornaros, M. E.; Paraskeva, C. A. Purification of olive mill wastewater phenols through membrane filtration and resin adsorption/desorption. J. Hazard. Mater. 2015, 285, 69-76.

(62) Dolaksiz, Y. E.; Temel, F.; Tabakci, M. Adsorption of phenolic compounds onto calix[4]arene-bonded silica gels from aqueous solutions. React. Funct. Polym. 2018, 126, 27-35.

(63) Zheng, Y. Z.; Chen, D. F.; Zhou, Y.; Guo, R.; Liang, Q.; Fu, Z. M. Hydrogen-bonding interactions between 1-butyl-2,3- dimethylimidazolium tetrafluoroborate and dimethyl sulphoxide. J. Mol. Struct. 2018, 1164, 70-76.

(64) Wu, X.; Yu, H.; Yuan, M.; Cao, H.; Ye, T.; Yu, J.; Xu, F. H-bond interactions between arsenite and deoxynucleotides at different $\mathrm{pH}$ values: A combined computational and experimental study. Chemosphere 2020, 251, 126395.

(65) Ahmad, M. N.; Karim, N. U.; Normaya, E.; Piah, B. M.; Iqbal, M. A. M.; Bulat, K. H. K. Artocarpus altilis extracts as a food-borne pathogen and oxidation inhibitors: RSM, COSMO RS, and molecular docking approaches. Sci. Rep. 2020, 10, 9566.

(66) Gil, M.; Louazil, P.; Iturmendi, N.; Moine, V.; Cheynier, V.; Saucier, C. Effect of polyvinylpolypyrrolidone treatment on rosés wines during fermentation: Impact on color, polyphenols and thiol aromas. Food Chem. 2019, 295, 493-498.

(67) Abadee, Z. G. N.; Hekmati, M.; Ganji, M. D. Removing phenol contaminants from wastewater using graphene nanobuds: DFT and reactive MD simulation investigations. J. Mol. Liq. 2019, 286, 110872.

(68) Aldred, E. M. Pharmacology: A handbook for complementary heathcare profesionals; first ed., Elsevier: Philadelphia, United State of America, 2008, 150-165.

(69) Zhou, H. Q.; Li, L.; Zhao, Y.; Wang, H. G.; Zheng, X. A combined experimental and density functional theory investigation of the hydrogen bonding of 2-cyclohexen-1-one and 3-methyl-2-cyclohexen1 -one in solvents. Spectrochim Acta Part A Mol. Biomol. Spectrosc. 2019, $208,32-39$.

(70) Beckee, A. D. A new mixing of Hartree-Fock and local densityfunctional theories. J. Chem. Phys. 1993, 98, 1372-1377.

(71) Kenawi, I. M.; Elnagdi, M. H. DFT and FT-IR analyses of hydrogen bonding in 3- substistuted-3-oxo-arylhydrazonopropanenitriles. Spectrochim Acta Part A Mol. Biomol. Spectrosc. 2006, 65, 805810.

(72) Normaya, E.; Saad, M. F. M.; Ahmad, M. N.; Bulat, K. H. K. COSMO-RS and DFT studies on development and optimization of quercetin as a chemosensor for $\mathrm{Fe} 3+$ recognition in aqueous medium. $J$. Mol. Struct. 2019, 1184, 538-545.

(73) Ting-ting, C.; Xing-wen, Z.; Mu-nan, Z.; Xu, J.; Chong-wei, C. Application of vacuum-ultraviolet (VUV) for phenolic homologues removal in humic acid solution: Efficiency, Pathway and DFT calculation. J. Hazard. Mater. 2019, 384, 121464. 